\title{
On Continuum Damage Modeling of Fiber Reinforced Viscoelastic Composites with Microcracks in terms of Invariants
}

\author{
Melek Usal \\ Department of Manufacturing Engineering, Süleyman Demirel University, 32260 Isparta, Turkey \\ Correspondence should be addressed to Melek Usal; melekusal@sdu.edu.tr
}

Received 30 December 2014; Accepted 23 February 2015

Academic Editor: Carla Roque

Copyright (C) 2015 Melek Usal. This is an open access article distributed under the Creative Commons Attribution License, which permits unrestricted use, distribution, and reproduction in any medium, provided the original work is properly cited.

\begin{abstract}
A continuum damage model is developed for the linear viscoelastic behavior of composites with microcracks consisting of an isotropic matrix reinforced by two arbitrarily independent and inextensible fiber families. Despite the fact that the matrix material is isotropic, the model in consideration bears the characteristic of directed media included in the transverse isotropy symmetry group solely due to its fibers distributions and the existence of microcracks. Using the basic laws of continuum damage mechanics and equations belonging to kinematics and deformation geometries of fibers, the constitutive functions have been obtained. It has been detected as a result of the thermodynamic constraints that the stress potential function is dependent on two symmetric tensors and two vectors, whereas the dissipative stress function is dependent on four symmetric tensors and two vectors. To determine arguments of the constitutive functionals, findings relating to the theory of invariants have been used as a method because of the fact that isotropy constraint is imposed on the material. As a result the linear constitutive equations of elastic stress, dissipative stress, and strain energy density release rate have been written in terms of material coordinate description. Using these expressions, total stress has been found.
\end{abstract}

\section{Introduction}

In various fields of industry, fiber reinforced composite materials are commonly used for load carrying components. In particular, these materials have become indispensable in advanced aerospace and aviation structures [1]. Fiber reinforced composites can display time dependent behavior (stress relaxation or creep) as a manifestation of one or both constituents deforming in a viscous manner [2]. Therefore, it is significant to develop a damage model that also includes the rate dependent nature of the viscoelastic material subjected to external loading. Damage modeling of composites is not an easy task, given the evolution of various damage mechanisms (matrix cracking, fiber breakage, interfacial debonding, transverse ply cracking, and ply delamination) in composite materials [3]. The effective stress theory assumes that the damage is uniformly distributed, where in fiber reinforced composites the complex damage occurs in a distributed manner with the damage mechanisms occurring in experimental work [1]. Some researches have conducted studies on the formulation of damage modeling on various composites [4-10].
Continuum damage mechanics (CDM), developed in the last few decades, provides a framework for incorporating the effects of damage induced stiffness softening, anisotropy, and so forth in constitutive equations [11]. Phenomenological continuum damage mechanics (CDM) models have been used extensively for modeling creep and fatigue damage by several authors as described in Lemaitre and Chaboche [12], Krajicinovic [13], and Voyiadjis and Kattan [14]. Chaboche has proposed a thermodynamic framework for continuum damage mechanics (CDM) for isotropic damage [15]. Furthermore, an updated continuum damage model was proposed, in which the fracture energy density, a function of the stress triaxiality, temperature, and strain rate in the transition region were taken as the critical damage factor [16]. Li et al. presented a damage model and its numerical solution by means of fast Fourier transforms (FFT) [17]. Much progress has been [18] made in the development of a consistent continuum framework for damage mechanics. Darabi et al. have proposed in their paper to enhance the continuum damage mechanics theories in modeling the microdamage healing 


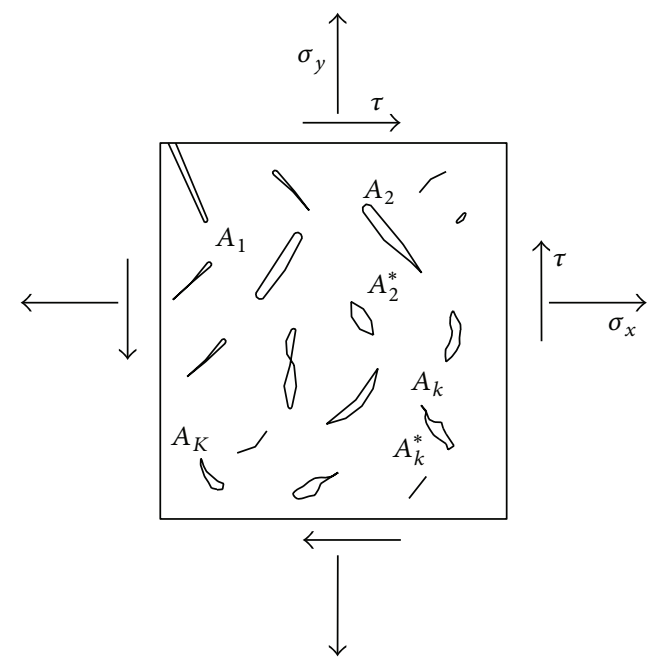

(a)

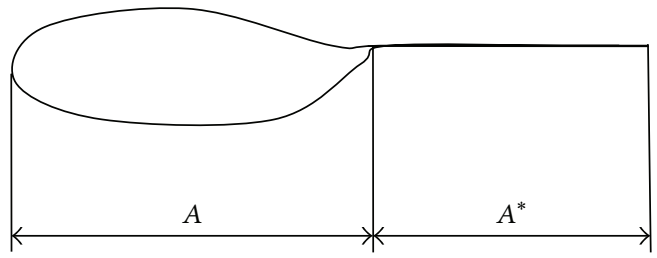

(b)

FIGURE 1: (a) A sketch of a representative volume element containing $N$ microcracks [21]. (b) Open and closed surfaces of a microcrack.

phenomenon in materials that tend to self-heal. This framework extends the well-known Kachanov's [19] effective configuration and the concept of the effective stress space to selfhealing materials by introducing the healing natural configuration in order to incorporate the microdamage healing effects [18].

Continuum damage mechanics $(\mathrm{CDM})$ has been proven to be a valuable tool for the determination of properties deterioration due to microcracks of composites. In continuum damage mechanics (CDM) models, families of damage are represented as continuous internal state variables, and concepts of irreversible thermodynamics are used in deriving constitutive equations [20]. A number of models of damage behavior of fiber reinforced composites are based on the methods of continuum damage mechanics (CDM). For the case of damage in viscoelastic materials two sets of internal variables are needed. Weitsman [21] presented a formulation to treat this case. In this formulation, damage in the form of microcracks is represented by two symmetric second rank tensors related to the total area of active and passive microcracks in a representative volume element (RVE) of the material and viscoelasticity is introduced through $n$ scalar valued internal state variables (ISVs). Since the scale of molecular rearrangement is much smaller than the scale of microcracks, the effect of damage on viscoelastic retardation time is ignored. The formulation uses Taylor series expansion of Gibbs free energy (Figure 1) [2].

Voyiadjis and Kattan [22] have presented a new formulation to link continuum damage mechanics with the concept of fabric tensors within the framework of classical elasticity assuming small strains. The new theory was called damage mechanics with fabric tensors. A fourth-rank damage tensor was used and its exact relationship to the fabric tensors was illustrated. A [23] model of damage mechanics for directional data was formulated using fabric tensors [22]. Qiang et al. have described accurately by a fourth-order fabric tensor of the cracks within the framework of noninteraction approximation the compliance increment due to the presence of generally nonuniformly distributed cracks. They have expressed that the compliance increment can be approximated by the corresponding second-order fabric tensor [24]. Spencer has formulated constitutive equations for flow of fabric-reinforced composite materials which show viscous and viscoplastic response at the forming temperature [24,25].

The fabric tensor is a symmetric second rank tensor which characterizes the geometric arrangement of the porous material microstructure. In developing this result it is assumed that the matrix material of the porous elastic solid is isotropic and, thus, that the anisotropy of the porous elastic solid is determined by the fabric tensor [26]. Fabric tensor has proved to be an effective tool statistically characterizing directional data in a smooth and frame-indifferent form. Directional data arising from microscopic physics and mechanics can be summed up as tensor-valued orientation distribution functions [27]. The subject of fabric tensor has been studied by a number of different researchers [28-31]. There are studies that establish a relationship between the fabric tensor with damage tensor. However, such studies are based on experimental data. In these studies, statistical distribution along a certain plane of the microcracks in the material has been considered and an average density of microcracks has been used. Our study is not based on experimental data and is phenomenological.

In this study in the scope of continuum damage mechanics $(\mathrm{CDM})$, considering representative volume element (RVE), the mechanical representation of damage was expressed with two interior conditional variables showing the properties of a second degree symmetrical tensor. It is assumed that an element from two different continuous fiber families is placed on each point of the composite material. In the scope of continuum mechanics, balance equations have been summarized and the combined form was stated 
for the energy equation and entropy inequality. Considering necessary constitutive axioms, after determining the arguments affecting the stress potential it was further proceeded to the formulation of the constitutive theory and a model has been formed for the damaged viscoelastic composite with isotropic matrix material. Considering fully orthogonal transformations for the material coordinate system, after determining the common invariants affecting both the stress potential and the dissipative stress, constitutive equations have been obtained related with the "elastic stress," the "dissipative stress," and the "strain energy density release rate."

On the other hand, inextensibility of the fibers and incompressibility of the composite are acceptable as broad recognition in practice in terms of formulation. Due to some technological requirements, it is aspired that specific construction elements have rather elastic properties, provided that they have high durability in certain directions. Fiberreinforced composite materials are produced sticking fibers in a polymeric matrix which is elastic but with low strength. These fibers are manufactured from high strength graphite or bor. They can be easily bent due to the very small size of their cross section. Assume that inextensibility of the fibers is a reasonable approach since the rigidity of the fibers is very high according to the rigidity of the matrix [32].

The constitutive model developed in this study is applicable for natural or biological fiber composites, such as living tissues, or synthetic composites for implant applications, because the incompressible (nearly incompressible) behavior of composites is often used for natural or biological fiber composites, such as living tissues, or synthetic composites for implant applications. In our study [33], we are concerned with developing the continuum damage mechanics model for elastic behavior of composites having microcracks consisting of an isotropic matrix reinforced by independent and inextensible two families of arbitrarily fibers.

\section{The Representation of Damage}

In some researches, in order to be able to define the damage variable, a representative volume element (RVE) has been considered that has a $k$ number of microcracks. While the open or active part of any $k$ th microcrack has been shown by $\mathbf{A}^{(k)}$, its closed or passive surface has been shown by $\mathbf{A}^{*(k)}$. Active or passive surfaces of a crack can switch positions among each other depending on stress, temperature, and humidity percentage. Despite that, Weitsman states that these open and closed surfaces can be selected as independent variables characterizing the state of a material at a certain time range $[21,34]$.

Stress and strain at the macrolevel are average values over the representative volume element (RVE) volume. Infinitesimal deformations can also be considered among these macrovalues. To fully consider the behaviors of representative volume element (RVE), it is necessary to deal with a $k$ number of crack parameters representing $\mathbf{A}^{(k)}$ and $\mathbf{A}^{*(k)}$ (no sum on $k, k=1, \ldots, N)$ surfaces. Because the real shape of these surfaces is unknown on the mesoscale, assuming them to be equivalent plane surfaces, Weitsman represented them by vectors $\mathbf{A}^{(k)}=A^{(k)} \mathbf{n}^{(k)}$ and $\mathbf{A}^{*(k)}=A^{*(k)} \mathbf{n}^{(k)}$. Here, $\mathbf{n}^{(k)}$ stands for a unit normal vector of a microcrack surface [34]. However, since each microcrack possesses two equal and opposite surfaces, the description should not discriminate between $\left(+\mathbf{n}^{k}\right)$ and $\left(-\mathbf{n}^{k}\right)$, which is accomplished by choosing the dyadic product as follows to represent the $k$ th flaw. This choice follows an analogous approach by Spencer [35]:

$$
\begin{array}{cc}
\mathbf{H}^{(k)}=\mathbf{A}^{(k)} \otimes \mathbf{A}^{(k)}, & \mathbf{H}^{*(k)}=\mathbf{A}^{*(k)} \otimes \mathbf{A}^{*(k)} \text { or } \\
H_{i j}^{(k)}=A_{i}^{(k)} A_{j}^{(k)}, & H_{i j}^{*(k)}=A_{i}^{*(k)} A_{j}^{*(k)} .
\end{array}
$$

In view of the paucity of information regarding the positions and sizes of the individual microflaws, it is proposed to represent damage by the dyadic sums of all $\mathbf{H}^{(k)},(k=$ $1,2, \ldots, N)$ and $\mathbf{H}^{*(k)},(k=1,2, \ldots, N)$ contained in the representative volume element (RVE), namely, by

$$
\begin{gathered}
\mathbf{H}=\sum_{k=1}^{N} \mathbf{H}^{(k)}=\sum_{k=1}^{N} \mathbf{A}^{(k)} \otimes \mathbf{A}^{(k)}, \\
\mathbf{H}^{*}=\sum_{k=1}^{N} \mathbf{H}^{*(k)}=\sum_{k=1}^{N} \mathbf{A}^{*(k)} \otimes \mathbf{A}^{*(k)} .
\end{gathered}
$$

Obviously, $\mathbf{H}$ and $\mathbf{H}^{*}$ are symmetric, second rank tensors. As the constitutive variable, in this study we are going to deal with only one damage tensor taking into consideration only the effect of open microsurfaces. In this study, due to the existence of fiber distributions and microvoids in the material it is assumed that the material has gained directed medium characteristics, that is, an anisotropic structure has appeared due to the damage and the fibers. We assume that initially the material was isotropic and that the anisotropy is only caused by the dispersion of microvoids and fibers. For a medium like that, the role of material description vectors will be played by the vector $\mathbf{A}(\mathbf{X}, t)$ representing the mean values in the representative volume element (RVE) and the vector $\dot{\mathbf{A}}(\mathbf{X}, t)$ representing the change in time of the preceding vector. We believe that, by dividing these vectors by the area of any characteristic surface pertaining to representative volume element (RVE), we render them dimensionless.

On the other hand, because the material will not be able to detect the positive and negative sides of microvoid surfaces, we had previously specified that the dependence on vectors $\mathbf{A}(\mathbf{X}, t)$ and $\dot{\mathbf{A}}(\mathbf{X}, t)$ can be expressed by a product of tensors:

$$
\begin{gathered}
\mathbf{H} \equiv \mathbf{A} \otimes \mathbf{A} \Longrightarrow \dot{\mathbf{H}} \equiv \dot{\mathbf{A}} \otimes \mathbf{A}+\mathbf{A} \otimes \dot{\mathbf{A}} \text { or } \\
H_{K L} \equiv A_{K} A_{L} \Longrightarrow \dot{H}_{K L} \equiv \dot{A}_{K} A_{L}+A_{K} \dot{A}_{L} .
\end{gathered}
$$

\section{Kinematics of Fibers Deformation and Thermomechanic Balance Equations}

Fiber families are represented by continuous vector fields $\mathbf{B}(\mathbf{X})$ and $\mathbf{Z}(\mathbf{X})$ before deformation and by continuous vector 
fields $\mathbf{b}(\mathbf{x})$ and $\mathbf{z}(\mathbf{x})$ after deformation. Relationships given below are true for $B$ and $Z$-fiber families $[35,36]$ :

$$
\begin{gathered}
b_{k}=\lambda_{b}^{-1} x_{k, K} B_{K}, \quad \lambda_{b} \equiv\left(\frac{d l}{d L}\right)_{b}, \\
\lambda_{b}^{2}=C_{K L} B_{K} B_{L} \\
z_{k}=\lambda_{z}^{-1} x_{k, K} Z_{K}, \quad \lambda_{z} \equiv\left(\frac{d l}{d L}\right)_{z}, \\
\lambda_{z}^{2}=C_{K L} Z_{K} Z_{L},
\end{gathered}
$$

where $d L$ and $d l$ are, respectively, arc length of fiber before and after deformation, $B_{K}$ and $Z_{K}$ are fiber vector components before deformation, $b_{k}$ and $z_{k}$ are fiber vector components after deformation, $x_{k, K}=\partial x_{k} / \partial X_{K}$ is deformation gradient, $\lambda_{b}$ and $\lambda_{z}$ are rates of extension of fiber families, and $C_{K L}=x_{k, K} x_{k, L}$ is Green deformation tensor.

The local thermomechanical balance equations can be summarized as follows [32, 37]:

conservation of mass:

$$
\dot{\rho}+\rho v_{k, k}=0
$$

conservation of mass in material representation:

$$
\rho(\mathbf{x}, t)=\frac{\rho_{0}(\mathbf{X})}{J(\mathbf{x}, t)},
$$

balance of linear momentum:

$$
\rho \dot{v}_{p}=\rho f_{p}+t_{r p, r}
$$

balance of moment of momentum:

$$
\varepsilon_{k r p} t_{r p}=0, \quad t_{r p}=t_{p r},
$$

conservation of energy:

$$
\rho \dot{\varepsilon}=t_{k l} d_{k l}+q_{k, k}+\rho h
$$

second law of thermodynamics:

$$
\rho \theta \dot{\eta}-\nabla \cdot \mathbf{q}+\frac{1}{\theta} \mathbf{q} \cdot \nabla \theta-\rho h \geq 0 .
$$

Here, $\mathbf{v}$ stands for the velocity field in a continuous medium, $\rho_{0}$ for mass density before deformation, $\rho$ for mass density after deformation, $J \equiv \operatorname{det}\left[x_{k, K}\right]=\rho_{0} / \rho(\mathbf{x}, t)$ for Jacobian, $\dot{\mathbf{v}}$ for acceleration, $t_{l k}$ for stress tensor, $f_{k}$ for the mechanical volumetric force per unit of mass, $\varepsilon$ for internal energy density per unit of mass, $q_{k}$ for heat flux vector, $h$ for heat source per unit of mass, $\eta$ for entropy density per unit of mass, $\theta(\mathbf{X}, t)$ for the absolute temperature of a material point $X$ at a moment $t$, and $\varepsilon_{i j k}$ for permutation tensor.

\section{Thermodynamic Constraints and Modeling Constitutive Equations}

Local energy equation (9) is then suitably combined with the entropy inequality (10) and, using a Legendre transformation such as $\psi \equiv \varepsilon-\theta \eta$ for free energy, entropy inequality is obtained as follows in the material form:

$$
-\left(\dot{\Sigma}+\rho_{0} \dot{\theta} \eta\right)+\frac{1}{2} T_{K L} \dot{C}_{K L}+\frac{1}{\theta} \theta_{, K} Q_{K} \geq 0
$$

Terms relating to the new values have been provided as follows:

$$
\begin{aligned}
\Sigma & \equiv \rho_{0} \psi, \\
\dot{C}_{K L}=2 d_{k l} x_{k, K} x_{l, L} & \Longrightarrow d_{k l}=\frac{1}{2} \dot{C}_{K L} X_{K, k} X_{L, l}, \\
T_{K L} \equiv J X_{K, k} X_{L, l} t_{k l} & \Longrightarrow t_{k l}=J^{-1} x_{k, K} x_{l, L} T_{K L}, \\
Q_{K} \equiv J X_{K, k} q_{k} & \Longrightarrow q_{k}=J^{-1} x_{k, K} Q_{K}, \\
\theta_{, K}=x_{k, K} \theta_{, k} & \Longrightarrow \theta_{, k}=X_{K, k} \theta_{, K} .
\end{aligned}
$$

Here, $\Sigma$ stands for thermodynamic stress potential, $\psi$ for generalized free energy density, $d_{k l}$ for deformation (strain) rate tensor, $X_{K, k}=\partial X_{K} / \partial x_{k}$ for the deformation gradient of the reverse motion, $T_{K L}$ for the stress tensor on material coordinates, $Q_{K}$ for the heat flux vector on material coordinates, and $\theta_{, K}$ for the temperature gradient on material coordinates.

For the use of inequality (11), which is a general expression of entropy generation, we need to know on which independent variables the thermodynamic potential $\Sigma$ depends as well as the mechanism of such dependence. Based on the material selected, the arguments of $\Sigma$ and variables it depends on have been found using constitutive axioms. Using the results of the axioms of causality, determinism, objectivity, smooth neighborhood, and admissibility axioms $[32,37]$, the arguments for $\Sigma$ allowing two fiber families and viscoelastic structures, bearing cracks, and where these cracks are believed to be changing with time, exposed to thermomechanical loading without heat transfer can be summarized as follows:

$$
\begin{gathered}
\Sigma=\Sigma\left(C_{K L}, H_{K L}, B_{K}, Z_{K}, \theta\right), \\
\eta=-\frac{1}{\rho_{0}} \frac{\partial \Sigma}{\partial \theta}, \\
\varepsilon=\frac{1}{\rho_{0}}\left(\Sigma-\frac{\partial \Sigma}{\partial \theta} \theta\right), \\
Q_{K}=0 \\
{ }_{E} T_{K L} \equiv 2 \frac{\partial \Sigma}{\partial C_{K L}},
\end{gathered}
$$




$$
\begin{gathered}
\bar{Y}_{M N} \equiv-\frac{\partial \Sigma}{\partial H_{M N}}, \quad Y_{K L} \equiv-\bar{Y}_{K L}, \quad Y_{K L} \equiv \frac{\partial \Sigma}{\partial H_{K L}} \\
{ }_{D} T_{M N}={ }_{D} T_{M N}\left(C_{M N}, \dot{C}_{M N}, H_{M N}, \dot{H}_{M N}, B_{K}, Z_{K}, \theta\right) \\
\quad \frac{1}{2}{ }_{D} T_{M N} \dot{C}_{M N}-Y_{M N} \dot{H}_{M N} \geq 0 \\
{ }_{D} T_{K L}\left(C_{M N}, \mathbf{0}, H_{M N}, \mathbf{0}, B_{K}, Z_{K}, \theta\right)=\mathbf{0} \\
T_{K L} \equiv{ }_{E} T_{K L}+{ }_{D} T_{K L} .
\end{gathered}
$$

In expression (18), $Y_{K L}$ is called as the strain energy density release. The definition $Y_{K L} \equiv-\bar{Y}_{K L}$ is used in order to deal with a positive value. In expression (22), dissipative stress tensor ${ }_{D} T_{K L}$ was defined with expression (19). ${ }_{E} T_{K L}$ is considered as an energetic stress. All energetic stresses are not elastic stresses, because a part of the energetic stress occurring in the material is spent for the formation of microcracks. But in this study, ${ }_{E} T_{K L}$ is called the elastic stress and it was defined with expression (17).

It is expressly understood from (17) and (18) that the elastic stress and the strain energy density release rate are derived from the stress potential $\Sigma$. From (19) it is understood that the dissipative stress is obtained in form of a matrix whose arguments are known. In this case, open forms of $\Sigma$ and ${ }_{D} T_{K L}$, which are known as constitutive functions with clear arguments, should be found.

However, firstly we should consider the constraints imposed by the material symmetry axiom onto the material under consideration. Because the symmetry group of the material under consideration is the fully orthogonal group, property $\left[S_{K L}\right]^{-1}=\left[S_{K L}\right]^{T}$, det $\mathbf{S}= \pm 1$ is true for the symmetry operation $\left[S_{K L}\right]$. Therefore, each material point conversion matches an orientation of the material medium. Such conversion should, for every $\left[S_{K L}\right]$, be in the following form

$$
X_{K}^{\prime}=S_{K L} X_{L}, \quad X_{L}=S_{L K}^{T} X_{K}^{\prime}, \quad\left[S_{K L}\right]^{-1}=\left[S_{K L}\right]^{T}
$$

and leave constitutive functionals form invariant. Mathematically this means the validity of the following conversions:

$$
\begin{aligned}
\Sigma( & \left.S_{K P} S_{L R} C_{P R}, S_{K P} S_{L R} H_{P R}, S_{K L} B_{L}, S_{K L} Z_{L}, \theta\right) \\
= & \sum\left(C_{K L}, H_{K L}, B_{K}, Z_{K}, \theta\right), \\
{ }_{D} T_{K L} & \left(S_{J P} S_{N R} C_{P R}, S_{J P} S_{N R} \dot{C}_{P R}, S_{J P} S_{N R} H_{P R},\right. \\
& \left.S_{J P} S_{N R} \dot{H}_{P R}, S_{J P} B_{P}, S_{J P} Z_{P}, \theta\right) \\
= & S_{K P} S_{L R} T_{D} T_{P R}\left(C_{J N}, \dot{C}_{J N}, H_{J N}, \dot{H}_{J N}, B_{J}, Z_{J}, \theta\right) .
\end{aligned}
$$

On the other hand, both incompressibility of the medium and inextensibility of the fiber are broadly recognized in terms of formulation. Assuming the medium to be incompressible and the fiber to be inextensible, the following requirements should be satisfied [32]:

$$
\begin{gathered}
J=1 \text { or } \operatorname{det} \mathbf{C}=I I I=1 \\
\lambda_{b}^{2}=C_{K L} B_{K} B_{L}=1, \quad \lambda_{z}^{2}=C_{K L} Z_{K} Z_{L}=1 .
\end{gathered}
$$

Thus, the constitutive equation for the elastic stress is obtained as follows in material coordinates:

$$
{ }_{E} T_{K L}=-p C_{K L}^{-1}+\Gamma_{b} B_{K} B_{L}+\Gamma_{z} Z_{K} Z_{L}+2 \frac{\partial \Sigma}{\partial C_{K L}} .
$$

In this expression, $p, \Gamma_{b}$, and $\Gamma_{z}$ are Lagrange coefficients and are defined by field equations and boundary conditions. $C_{K L}^{-1} \equiv X_{K, l} X_{L, l}$ is the Piola deformation tensor.

\section{Determination of Elastic Stress and Strain Energy Density Release Rate Constitutive Equations}

Since the matrix has been assumed to be isotropic, relation (24) is expressed as follows;

$$
\begin{aligned}
& \sum\left(C_{K L}, H_{K L}, B_{K}, Z_{K}, \theta\right) \\
& \quad=\Sigma\left(M_{K P} M_{L R} C_{P R}, M_{K P} M_{L R} H_{P R}, M_{K L} B_{L}, M_{K L} Z_{L}, \theta\right) .
\end{aligned}
$$

Here, the orthogonal matrix indicating the symmetry group $\left\{M_{K L}\right\}$ will be expressed for $\left[M_{K L}\right] \in O(3)$ and property $\left[M_{K L}\right]^{-1}=\left[M_{K L}\right]^{T} \Longrightarrow \operatorname{det} \mathbf{M}= \pm 1$ is true.

On the other hand, since $\Sigma$ has been assumed to be the analytical function of its arguments, such arguments, which are expected to remain invariant under orthogonal transformations belonging to the symmetry group, should depend on a finite number of invariants:

$$
\begin{gathered}
I_{1} \equiv C_{K K}, \quad I_{2} \equiv C_{K L} C_{L K}, \\
I_{3} \equiv C_{K L} C_{L M} C_{M K}, \quad I_{4}=H_{K K}, \\
I_{5} \equiv B_{K} B_{K}, \quad I_{6} \equiv Z_{K} Z_{K}, \\
I_{7} \equiv B_{K} Z_{K}, \quad I_{8} \equiv B_{K} C_{K L} B_{L}=\lambda_{b}^{2}, \\
I_{9} \equiv B_{K} C_{K L} C_{L M} B_{M}, \quad I_{10} \equiv Z_{K} C_{K L} Z_{L}=\lambda_{z}^{2}, \\
I_{11} \equiv Z_{K} C_{K L} C_{L M} Z_{M}, \quad I_{12} \equiv B_{K} C_{K L} Z_{L}, \\
I_{13} \equiv B_{K} C_{K L} C_{L M} Z_{M}, \quad I_{14} \equiv B_{K} H_{K L} B_{L}, \\
I_{15} \equiv B_{K} C_{K L} H_{L M} B_{M}, \quad I_{16} \equiv Z_{K} H_{K L} Z_{L}, \\
I_{17} \equiv Z_{K} C_{K L} H_{L M} Z_{M}, \quad I_{18} \equiv B_{K} H_{K L} Z_{L}, \\
I_{19} \equiv B_{K} C_{K L} H_{L M} Z_{M}, \quad I_{20} \equiv C_{K L} H_{L K}, \\
I_{21} \equiv C_{K L} C_{L M} H_{M K} .
\end{gathered}
$$


Instead of the first three invariants of the Green deformation tensor $\mathbf{C}$, we can use the principal invariants as follows:

$$
\begin{gathered}
I=I_{1}, \quad I I=\frac{1}{2}\left(I_{1}^{2}-I_{2}\right), \\
I I I=\frac{1}{6}\left(I_{1}^{3}-3 I_{1} I_{2}+2 I_{3}\right)=\operatorname{det} \mathbf{C} .
\end{gathered}
$$

Given the incompressibility of the composite, inextensibility of the fiber families, and the fact that $\mathbf{B}$ and $\mathbf{Z}$ are unit vectors, the invariants $I I I, I_{8}, I_{10}, I_{5}$, and $I_{6}$ in expressions (29) and (30) are equal to 1 thus eliminating the dependence of $\Sigma$ on these invariants. As a result, the invariants on which $\Sigma$ depends are expressed as follows:

$$
\Sigma=\Sigma\left(I, I I, I_{4}, I_{7}, I_{9}, I_{m}, \theta\right) \quad(m=11, \ldots, 21) .
$$

Taking the derivative of expression (31) according to $C_{P R}$ and $H_{P R}$ and substituting it into (27) and (18), the following expressions are obtained:

$$
\begin{aligned}
{ }_{E} T_{P R}= & -p C_{P R}^{-1}+\Gamma_{b} B_{P} B_{R}+\Gamma_{z} Z_{P} Z_{R} \\
+ & 2\left(\frac{\partial \Sigma}{\partial I} \frac{\partial I}{\partial C_{P R}}+\frac{\partial \Sigma}{\partial I I} \frac{\partial I I}{\partial C_{P R}}+\frac{\partial \Sigma}{\partial I_{i}} \frac{\partial I_{i}}{\partial C_{P R}}\right), \\
& (i=9,11,12,13,15,17,19,20,21) \\
Y_{P R}= & \frac{\partial \Sigma}{\partial I_{m}} \frac{\partial I_{m}}{\partial H_{P R}} \quad(m=4,14,15, \ldots, 21) .
\end{aligned}
$$

It is understood that, as always, repeated indices will undergo summation. If derivatives of invariants appearing in these equations according to $C_{P R}$ and $H_{P R}$ are taken from expressions (29) and (30) and substituted afterwards, constitutive equation of the elastic stress in nonlinear form is obtained as follows:

$$
\begin{aligned}
{ }_{E} T_{P R}= & -p C_{P R}^{-1}+\Gamma_{b} B_{P} B_{R}+\Gamma_{z} Z_{P} Z_{R} \\
& +2\left\{\left(\frac{\partial \Sigma}{\partial I}+\frac{\partial \Sigma}{\partial I I} C_{L L}\right) \delta_{P R}-\frac{\partial \Sigma}{\partial I I} C_{P R}\right. \\
& +\frac{\partial \Sigma}{\partial I_{9}}\left(B_{P} B_{L} C_{L R}+C_{P L} B_{L} B_{R}\right) \\
& +\frac{\partial \Sigma}{\partial I_{11}}\left(Z_{P} Z_{L} C_{L R}+C_{P L} Z_{L} Z_{R}\right) \\
& +\frac{\partial \Sigma}{\partial I_{12}} B_{P} Z_{R} \\
& +\frac{\partial \Sigma}{\partial I_{13}}\left(B_{P} Z_{L} C_{L R}+C_{P L} B_{L} Z_{R}\right)
\end{aligned}
$$

$$
\begin{aligned}
& +\frac{\partial \Sigma}{\partial I_{15}}\left(B_{P} B_{L} H_{L R}+H_{P L} B_{L} B_{R}\right) \\
& +\frac{\partial \Sigma}{\partial I_{17}}\left(Z_{P} Z_{L} H_{L R}+H_{P L} Z_{L} Z_{R}\right) \\
& +\frac{\partial \Sigma}{\partial I_{19}}\left(B_{P} Z_{L} H_{L R}+H_{P L} B_{L} Z_{R}\right) \\
& \left.+\frac{\partial \Sigma}{\partial I_{20}} H_{P R}+\frac{\partial \Sigma}{\partial I_{21}}\left(C_{P L} H_{L R}+H_{P L} C_{L R}\right)\right\} .
\end{aligned}
$$

And the strain energy density release rate in nonlinear form is obtained as follows:

$$
\begin{aligned}
Y_{P R}= & \frac{\partial \Sigma}{\partial I_{4}} \delta_{P R}+\frac{\partial \Sigma}{\partial I_{14}} B_{P} B_{R}+\frac{\partial \Sigma}{\partial I_{15}} C_{P K} B_{K} B_{R} \\
& +\frac{\partial \Sigma}{\partial I_{16}} Z_{P} Z_{R}+\frac{\partial \Sigma}{\partial I_{17}} C_{P L} Z_{L} Z_{R}+\frac{\partial \Sigma}{\partial I_{18}} B_{P} Z_{R} \\
& +\frac{\partial \Sigma}{\partial I_{19}} C_{P L} B_{L} Z_{R}+\frac{\partial \Sigma}{\partial I_{20}} C_{P R}+\frac{\partial \Sigma}{\partial I_{21}} C_{P L} C_{L R}
\end{aligned}
$$

More concrete form of the constitutive equations given by (33) and (34) can be obtained provided that Lagrange coefficients $-p, \Gamma_{b}$, and $\Gamma_{z}$ and the derivatives of $\Sigma$ based on its invariants are known. It has been already stated that $-p, \Gamma_{b}$, and $\Gamma_{z}$ can be obtained from field equations and boundary conditions. To obtain the derivatives of $\Sigma$ according to its invariants, how $\Sigma$ depends on the invariants it is shown to depend on in expression (31) should be estimated. In this study, the matrix material has been considered as an isotropic medium. According to the fact that $\sum$ is an analytical function of those invariants, assuming that this function is analytic, the stress potential is expanded in the power series around natural condition. To obtain a quadratic theory, the terms in this series expanding should be kept to second order; therefore, the stress potential can be represented by a polynomial $[32,37]$. However, the grade and number of terms of the polynomial representing $\Sigma$ depend on the size of its deformation invariant and their shares of interaction in the case [38-40].

In this study, mechanical interactions and effect of damage have been assumed to be linear. Furthermore, considering that the material remains insensitive to directional changes along fibers, double components of fiber vectors have been included in the operation. Because mechanical interactions and effect of damage are assumed to be linear, the stress should remain linear according to the deformation tensor and the damage tensor. Therefore, function $\Sigma$ could be represented by a second degree polynomial according to the invariants it depends on. On the other hand, because internal energy is defined as a positive definite form, for a polynomial to be positively defined and for the order of invariants not to affect $\Sigma$, the polynomial must have symmetric coefficients; that is, it must be in a quadratic form. Accordingly, if polynomial approximation is selected, the following expression can 
be recorded for the stress potential $\Sigma$ in terms of the existing invariants:

$$
\Sigma=\Sigma_{i . j} a_{i j} I_{i} I_{j}, \quad(i, j=1,2,4,7,9,11, \ldots, 21), a_{i j}=a_{j i} .
$$

The derivatives of $\Sigma$ based on its invariants in (33) and (34) are obtained from expression (35) as follows:

$$
\begin{gathered}
\frac{\partial \Sigma}{\partial I}=2\left(a_{1,1} I+a_{1,2} I I+a_{1, k} I_{k}\right), \\
\frac{\partial \Sigma}{\partial I I}=2\left(a_{1,2} I+a_{2,2} I I+a_{2, k} I_{k}\right), \\
\frac{\partial \Sigma}{\partial I_{m}}=2\left(a_{m, 1} I+a_{m, 2} I I+a_{m, k} I_{k}\right), \\
(m=4,9,11, \ldots, 21), \quad(k=4,7,9,11, \ldots, 21) .
\end{gathered}
$$

At this stage, expressions (29) and (30) have shown on what the invariants in expression (36) depend. Due to the existence of the relationship $C_{K L}=\delta_{K L}+2 E_{K L}$ between the Green deformation tensor and the strain tensor and assuming that mechanic interactions are linear $\left(E_{K L} \cong \widetilde{E}_{K L}=\right.$ $\left.(1 / 2)\left(U_{K, L}+U_{L, K}\right)\right)$, those invariants that depend on the Green deformation tensor $\left(C_{K L}\right)$ have been expressed in terms of strain tensor $\left(E_{K L}\right)$, which is a more useful parameter.

Terms after the third term on the right side of (33) and all terms of the right side of (34) have been calculated using the partial derivatives given in expression (36) and invariants that depend on the strain tensor $\left(E_{K L}\right)$. Due to the assumptions made in this study, of the first grade components of the strain tensor $E_{K L}$ and the damage tensor $H_{K L}$ and of the external multiplication components of vectors $B_{K}$ and $Z_{K}$, those whose number is even have been taken into consideration. Thus, in the beginning, the elastic stress is expressed for the condition without stress and without load (with the term $\alpha_{1} \delta_{P R}$ assumed to be zero) by taking common coefficients into common parenthesis:

$$
\begin{aligned}
{ }_{E} T_{P R}= & -p C_{P R}^{-1}+\Gamma_{b} B_{P} B_{R}+\Gamma_{z} Z_{P} Z_{R}+\alpha_{2} E_{K K} \delta_{P R} \\
& +\alpha_{3} H_{K K} \delta_{P R}+\alpha_{4} B_{K} H_{K N} B_{N} \delta_{P R} \\
& +\alpha_{5} Z_{K} H_{K N} Z_{N} \delta_{P R}+\alpha_{6} E_{P R}+\alpha_{7} E_{K K} B_{P} B_{R} \\
& +\alpha_{8} H_{K K} B_{P} B_{R}+\alpha_{9} Z_{K} H_{K N} Z_{N} B_{P} B_{R} \\
& +\alpha_{10}\left[B_{P} B_{R}+\left(B_{P} B_{L} E_{L R}+E_{P L} B_{L} B_{R}\right)\right] \\
& +\alpha_{11} E_{K K} Z_{P} Z_{R}+\alpha_{12} H_{K K} Z_{P} Z_{R} \\
& +\alpha_{13} B_{K} H_{K N} B_{N} Z_{P} Z_{R} \\
& +\alpha_{14}\left[Z_{P} Z_{R}+\left(Z_{P} Z_{L} E_{L R}+E_{P L} Z_{L} Z_{R}\right)\right] \\
& +\alpha_{15} B_{K} Z_{K} B_{P} Z_{R}+\alpha_{16} B_{K} E_{K N} Z_{N} B_{P} Z_{R} \\
& +\alpha_{17} B_{K} H_{K N} Z_{N} B_{P} Z_{R}
\end{aligned}
$$

$$
\begin{aligned}
& +\alpha_{18} B_{K} Z_{K}\left(B_{P} Z_{L} E_{L R}+E_{P L} B_{L} Z_{R}\right) \\
& +\alpha_{19}\left(B_{P} B_{L} H_{L R}+H_{P L} B_{L} B_{R}\right) \\
& +\alpha_{20}\left(Z_{P} Z_{L} H_{L R}+H_{P L} Z_{L} Z_{R}\right) \\
& +\alpha_{21} B_{K} Z_{K}\left(B_{P} Z_{L} H_{L R}+H_{P L} B_{L} Z_{R}\right)+\alpha_{22} H_{P R}
\end{aligned}
$$

The strain energy density release rate is expressed as follows by taking common coefficients into parenthesis in the beginning, without microcracks (the term $\gamma_{1} \delta_{P R}$ is taken here as zero) being obtained as follows:

$$
\begin{aligned}
Y_{P R}= & \gamma_{2} E_{K K} \delta_{P R}+\gamma_{3} H_{K K} \delta_{P R}+\gamma_{4} B_{K} H_{K N} B_{N} \delta_{P R} \\
& +\gamma_{5} Z_{K} H_{K N} Z_{N} \delta_{P R}+\gamma_{6} B_{P} B_{R}+\gamma_{7} E_{K K} B_{P} B_{R} \\
& +\gamma_{8} H_{K K} B_{P} B_{R}+\gamma_{9} Z_{K} H_{K N} Z_{N} B_{P} B_{R}+\gamma_{10} E_{P N} B_{N} B_{R} \\
& +\gamma_{11} Z_{P} Z_{R}+\gamma_{12} E_{K K} Z_{P} Z_{R}+\gamma_{13} H_{K K} Z_{P} Z_{R} \\
& +\gamma_{14} B_{K} H_{K N} B_{N} Z_{P} Z_{R}+\gamma_{15} E_{P N} Z_{N} Z_{R} \\
& +\gamma_{16} B_{K} Z_{K} B_{P} Z_{R}+\gamma_{17} B_{K} E_{K N} Z_{N} B_{P} Z_{R} \\
& +\gamma_{18} B_{K} H_{K N} Z_{N} B_{P} Z_{R}+\gamma_{19} B_{K} Z_{K} E_{P N} B_{N} Z_{R}+\gamma_{20} E_{P R} .
\end{aligned}
$$

Coefficients $\left[\alpha_{k}(k=1,2,3, \ldots, 22)\right.$ and $\gamma_{k}(k=1,2$, $3, \ldots, 20)]$ in (37) and (38) have been depending on the medium temperature $\theta$ and $a_{i j}$.

In a composite material that consists of an isotropic matrix reinforced by two arbitrary independent and inextensible fiber families, the medium is assumed to be incompressible and homogeneous, has microcracks, and shows linear viscoelastic behavior. Equation (37) is the linear constitutive equation of elastic stress. First, second and third terms of (37) are hydrostatic pressure and contributions of fiber tensions to the elastic stress, respectively; fourth and eighth terms combined are the contribution of the elastic deformation; fifth and twenty sixth terms combined are the contribution of the damage tensor; sixth, tenth, and twenty-third terms are the stress arising of the interaction between the fiber distribution $\mathbf{B}$ and the damage tensor; seventh, fifteenth, and twenty-fourth terms are the stress arising of the interaction between the fiber distribution $\mathbf{Z}$ and the damage tensor; ninth and thirteenth terms are the stress arising of the interaction between the fiber distribution $\mathbf{B}$ and the elastic deformation; eleventh, sixteenth, twenty-first, and twenty-fifth terms are the contribution produced by the triple interaction between the fiber fields $\mathbf{B}$ and $\mathbf{Z}$ and the damage tensor; twelfth term is the contribution of fiber distribution $\mathbf{B}$; fourteenth and eighteenth terms are the stress arising of the interaction between the fiber distribution $\mathbf{Z}$ and the elastic deformation; seventeenth term is the contribution of fiber distribution $\mathbf{Z}$; nineteenth term is the stress produced by the interaction between the fiber field $\mathbf{B}$ and the fiber field $\mathbf{Z}$; and twentieth and twenty second terms are the contribution produced by the triple interaction between the fiber fields $\mathbf{B}$ and $\mathbf{Z}$ and the elastic deformation field. 
Equation (38) is the linear constitutive equation of strain energy density release rate. First and nineteenth terms combined are the contribution of the elastic deformation; second term is the contribution of the damage tensor; third and seventh terms combined are the strain energy density release arising of the interaction between the fiber distribution $\mathbf{B}$ and the damage tensor; fourth and twelfth terms are the strain energy density release arising of the interaction between the fiber distribution $\mathbf{Z}$ and the damage tensor; fifth term is the contribution of fiber distribution $\mathbf{B}$; sixth and ninth terms are the strain energy density release arising of the interaction between the fiber distribution $\mathbf{B}$ and the elastic deformation; eighth, thirteenth, and seventeenth terms are the contribution produced by the triple interaction between the fiber fields $\mathbf{B}$ and $\mathbf{Z}$ and the damage tensor; tenth term is the contribution of fiber distribution $\mathbf{Z}$; eleventh and fourteenth terms are the strain energy density release arising of the interaction between the fiber distribution $\mathbf{Z}$ and the elastic deformation; fifteenth term is the strain energy density release produced by the interaction between the fiber field $\mathbf{B}$ and the fiber field $\mathbf{Z}$; and sixteenth and eighteenth terms are the contribution produced by the triple interaction between the fiber fields $\mathbf{B}$ and $\mathbf{Z}$ and the elastic deformation field.

\section{Determination of Dissipative Stress Constitutive Equation}

It is assumed that the viscoelastic behavior of the medium in consideration is in conformity with Kelvin-Voigt model. It has been determined that the dissipative stress depends on deformation, deformation rate, damage, damage rate, and distributions of fibers, yielding in expressions (19)-(21). Additional constraints imposed on the dissipative stress by constitutive functions originate from the material symmetry of the medium. The structure of the dissipative stress should be in compliance with the following transformation for each orthogonal matrix $\left[M_{K L}\right] \in O(3)$ belonging to the symmetry group of the material:

$$
\begin{gathered}
{ }_{D} T_{K L}\left(M_{J P} M_{N R} C_{P R}, M_{J P} M_{N R} \dot{C}_{P R}, M_{J P} M_{N R} H_{P R},\right. \\
\left.M_{J P} M_{N R} \dot{H}_{P R}, M_{J P} B_{P}, M_{J P} Z_{P}, \theta\right) \\
=M_{K P} M_{L R D} T_{P R}\left(C_{J N}, \dot{C}_{J N}, H_{J N}, \dot{H}_{J N}, B_{J}, Z_{J}, \theta\right),
\end{gathered}
$$

where the matrix is isotropic; relation (39) is valid for each orthogonal matrix of the fully orthogonal group. The dissipative stress is an isotropic function of the symmetric matrices $C_{K L}, \dot{C}_{K L}, H_{K L}, \dot{H}_{K L}$ and polar vectors $B_{K}$ and $Z_{K}$. For simplicity of notation, dependence of the dissipative stress tensor on $\theta$ has not been denoted. To obtain the explicit expression of the tensor component ${ }_{D} T_{K L}$ in terms of its invariant arguments, the following way has been followed according to the theory of invariants [41]: $V_{K}$ is an arbitrary vector, ${ }_{D} T_{K L}$ and the vector $V_{K}$ are multiplied on the right and on the left by scalar multiplication, and the product is defined by a scalar function $\mathfrak{R}$.

\section{Consider}

$$
\begin{aligned}
\Re & \left(C_{K L}, \dot{C}_{K L}, H_{K L}, \dot{H}_{K L}, B_{K}, Z_{K}, V_{K}\right) \\
& \equiv V_{K} V_{L D} T_{K L}\left(C_{P R}, \dot{C}_{P R}, H_{P R}, \dot{H}_{P R}, B_{P}, Z_{P}\right) .
\end{aligned}
$$

Here the $\Re$ scalar is an isotropic function of the symmetric matrices $C_{K L}, \dot{C}_{K L}, H_{K L}, \dot{H}_{K L}$ and absolute vectors $B_{K}, Z_{K}$, and $V_{K}$. Taking the partial derivative of expression (40) according to $V_{K}$ and $V_{L}$, the following expression can be recorded [32]:

$$
\begin{aligned}
{ }_{D} T_{K L}\left(C_{P R}, \dot{C}_{P R}, H_{P R}, \dot{H}_{P R}, B_{P}, Z_{P}\right) \\
\quad=\frac{1}{2} \frac{\partial^{2} \Re\left(C_{K L}, \dot{C}_{K L}, H_{K L}, \dot{H}_{K L}, B_{K}, Z_{K}, V_{K}\right)}{\partial V_{K} \partial V_{L}} \mid .
\end{aligned}
$$

Because the left side of this expression is independent from the vector $\mathbf{V}, \mathbf{V}=\mathbf{0}$ should be true for inequality (41). In this situation, isotropic tensor function ${ }_{D} \bar{T}_{K L}$ is expressed as follows:

$$
\begin{aligned}
& { }_{D} T_{K L}\left(C_{P R}, \dot{C}_{P R}, H_{P R}, \dot{H}_{P R}, B_{P}, Z_{P}\right) \\
& \quad=\left.\frac{1}{2} \frac{\partial^{2} \Re\left(C_{K L}, \dot{C}_{K L}, H_{K L}, \dot{H}_{K L}, B_{K}, Z_{K}, V_{K}\right)}{\partial V_{K} \partial V_{L}}\right|_{V_{K}=0} .
\end{aligned}
$$

In this situation, to find the tensor ${ }_{D} T_{K L}$ from relationship (42), structure of the scalar $\mathfrak{R}$ should be determined that depends on the arguments $C_{K L}, \dot{C}_{K L}, H_{K L}, \dot{H}_{K L}, B_{K}, Z_{K}, V_{K}$ and second degree partial derivative of this function according to the vector $\mathbf{V}$ should be calculated at $\mathbf{V}=\mathbf{0}$. Let us first remove the arbitrary vector $\mathbf{V}$ from the arguments of the scalar function $\mathfrak{R}$ and define a scalar function with arguments $C_{K L}, \dot{C}_{K L}, H_{K L}, \dot{H}_{K L}, B_{K}, Z_{K}$ :

$$
\Xi \equiv \Xi\left(C_{K L}, \dot{C}_{K L}, H_{K L}, \dot{H}_{K L}, B_{K}, Z_{K}\right)
$$

For $\Xi$, which is an isotropic function, to keep the invariant under orthogonal coordinate transformations, its arguments must depend on a finite number of invariants. Using the methods in the theory of invariants [41], 98 invariants of the four symmetric tensors $C_{K L}, \dot{C}_{K L}, H_{K L}, \dot{H}_{K L}$ and the two polar vectors $B_{K}, Z_{K}$, which are independent of each other, have been expressed in the following list:

$$
\begin{gathered}
I_{1} \equiv C_{K K}, \quad I_{2} \equiv C_{K L} C_{L K}, \\
I_{3} \equiv C_{K L} C_{L M} C_{M K}, \quad I_{4} \equiv \dot{C}_{K K}, \\
I_{5} \equiv \dot{C}_{K L} \dot{C}_{L K}, \quad I_{6} \equiv \dot{C}_{K L} \dot{C}_{L M} \dot{C}_{M K}, \\
I_{7} \equiv H_{K K}, \quad I_{8} \equiv \dot{H}_{K K},
\end{gathered}
$$




$$
\begin{aligned}
& I_{9} \equiv C_{K L} \dot{C}_{L K}, \quad I_{10} \equiv C_{K L} H_{L K}, \\
& I_{11} \equiv C_{K L} \dot{H}_{L K}, \quad I_{12} \equiv \dot{C}_{K L} H_{L K}, \\
& I_{13} \equiv \dot{C}_{K L} \dot{H}_{L K}, \quad I_{14} \equiv H_{K L} \dot{H}_{L K}, \\
& I_{15} \equiv C_{K L} C_{L M} \dot{C}_{M K}, \quad I_{16} \equiv C_{K L} C_{L M} H_{M K} \text {, } \\
& I_{17} \equiv C_{K L} C_{L M} \dot{H}_{M K}, \quad I_{18} \equiv C_{K L} \dot{C}_{L M} \dot{C}_{M K}, \\
& I_{19} \equiv \dot{C}_{K L} \dot{C}_{L M} H_{M K}, \quad I_{20} \equiv \dot{C}_{K L} \dot{C}_{L M} \dot{H}_{M K} \text {, } \\
& I_{21} \equiv C_{K L} \dot{C}_{L M} H_{M K}, \quad I_{22} \equiv C_{K L} \dot{C}_{L M} \dot{H}_{M K} \text {, } \\
& I_{23} \equiv C_{K L} H_{L M} \dot{H}_{M K}, \quad I_{24} \equiv C_{K L} \dot{H}_{L M} \dot{H}_{M K} \text {, } \\
& I_{25} \equiv C_{K L} C_{L M} \dot{C}_{M N} H_{N K}, \quad I_{26} \equiv C_{K L} C_{L M} \dot{C}_{M N} \dot{H}_{N K} \text {, } \\
& I_{27} \equiv C_{K L} C_{L M} H_{M N} \dot{H}_{N K}, \quad I_{28} \equiv \dot{C}_{K L} \dot{C}_{L M} C_{M N} H_{N K} \text {, } \\
& I_{29} \equiv \dot{C}_{K L} \dot{C}_{L M} C_{M N} \dot{H}_{N K}, \quad I_{30} \equiv \dot{C}_{K L} \dot{C}_{L M} H_{M N} \dot{H}_{N K} \text {, } \\
& I_{31} \equiv C_{K L} C_{L M} \dot{C}_{M N} \dot{C}_{N K}, \quad I_{32} \equiv C_{K L} \dot{C}_{L M} H_{M N} \dot{H}_{N K} \text {, } \\
& I_{33} \equiv B_{K} B_{K}, \quad I_{34} \equiv Z_{K} Z_{K}, \\
& I_{35} \equiv B_{K} Z_{K}, \quad I_{36} \equiv B_{K} C_{K L} B_{L}=\lambda_{b}^{2}, \\
& I_{37} \equiv B_{K} C_{K L} C_{L M} B_{M}, \quad I_{38} \equiv B_{K} \dot{C}_{K L} B_{L} \text {, } \\
& I_{39} \equiv B_{K} \dot{C}_{K L} \dot{C}_{L M} B_{M}, \quad I_{40} \equiv B_{K} H_{K L} B_{L}, \\
& I_{41} \equiv B_{K} \dot{H}_{K L} B_{L}, \quad I_{42} \equiv B_{K} C_{K L} \dot{C}_{L M} B_{M}, \\
& I_{43} \equiv B_{K} C_{K L} H_{L M} B_{M}, \quad I_{44} \equiv B_{K} C_{K L} \dot{H}_{L M} B_{M} \text {, } \\
& I_{45} \equiv B_{K} \dot{C}_{K L} H_{L M} B_{M}, \quad I_{46} \equiv B_{K} \dot{C}_{K L} \dot{H}_{L M} B_{M} \text {, } \\
& I_{47} \equiv B_{K} H_{K L} \dot{H}_{L M} B_{M}, \\
& I_{48} \equiv B_{K} C_{K L} \dot{C}_{L M} H_{M N} B_{N} \text {, } \\
& I_{49} \equiv B_{K} C_{K L} \dot{C}_{L M} \dot{H}_{M N} B_{N} \text {, } \\
& I_{50} \equiv B_{K} C_{K L} H_{L M} \dot{H}_{M N} B_{N} \text {, } \\
& I_{51} \equiv B_{K} \dot{C}_{K L} H_{L M} \dot{H}_{M N} B_{N} \text {, } \\
& I_{52} \equiv B_{K} C_{K L} C_{L M} \dot{C}_{M N} H_{N S} B_{S} \text {, } \\
& I_{53} \equiv B_{K} \dot{C}_{K L} \dot{C}_{L M} H_{M N} C_{N S} B_{S} \text {, } \\
& I_{54} \equiv B_{K} C_{K L} C_{L M} \dot{C}_{M N} \dot{H}_{N S} B_{S} \text {, } \\
& I_{55} \equiv B_{K} \dot{C}_{K L} \dot{C}_{L M} H_{M N} \dot{H}_{N S} B_{S}, \\
& I_{56} \equiv B_{K} C_{K L} \dot{C}_{L M} H_{M N} \dot{H}_{N S} B_{S} \text {, } \\
& I_{57} \equiv Z_{K} C_{K L} Z_{L}=\lambda_{z}^{2} \text {, } \\
& I_{58} \equiv Z_{K} C_{K L} C_{L M} Z_{M} \text {, } \\
& I_{59} \equiv Z_{K} \dot{C}_{K L} Z_{L}, \quad I_{60} \equiv Z_{K} \dot{C}_{K L} \dot{C}_{L M} Z_{M} \text {, }
\end{aligned}
$$

$$
\begin{aligned}
& I_{61} \equiv Z_{K} H_{K L} Z_{L}, \quad I_{62} \equiv Z_{K} \dot{H}_{K L} Z_{L}, \\
& I_{63} \equiv Z_{K} C_{K L} \dot{C}_{L M} Z_{M}, \quad I_{64} \equiv Z_{K} C_{K L} H_{L M} Z_{M} \text {, } \\
& I_{65} \equiv Z_{K} C_{K L} \dot{H}_{L M} Z_{M}, \quad I_{66} \equiv Z_{K} \dot{C}_{K L} H_{L M} Z_{M} \text {, } \\
& I_{67} \equiv Z_{K} \dot{C}_{K L} \dot{H}_{L M} Z_{M}, \quad I_{68} \equiv Z_{K} H_{K L} \dot{H}_{L M} Z_{M} \text {, } \\
& I_{69} \equiv Z_{K} C_{K L} \dot{C}_{L M} H_{M N} Z_{N} \text {, } \\
& I_{70} \equiv Z_{K} C_{K L} \dot{C}_{L M} \dot{H}_{M N} Z_{N} \text {, } \\
& I_{71} \equiv Z_{K} C_{K L} H_{L M} \dot{H}_{M N} Z_{N} \text {, } \\
& I_{72} \equiv Z_{K} \dot{C}_{K L} H_{L M} \dot{H}_{M N} Z_{N} \text {, } \\
& I_{73} \equiv Z_{K} C_{K L} C_{L M} \dot{C}_{M N} H_{N S} Z_{S} \text {, } \\
& I_{74} \equiv Z_{K} C_{K L} C_{L M} \dot{C}_{M N} \dot{H}_{N S} Z_{S} \text {, } \\
& I_{75} \equiv Z_{K} \dot{C}_{K L} \dot{C}_{L M} H_{M N} C_{N S} Z_{S} \text {, } \\
& I_{76} \equiv Z_{K} \dot{C}_{K L} \dot{C}_{L M} H_{M N} \dot{H}_{N S} Z_{S} \text {, } \\
& I_{77} \equiv Z_{K} C_{K L} \dot{C}_{L M} H_{M N} \dot{H}_{N S} Z_{S} \text {, } \\
& I_{78} \equiv B_{K} C_{K L} Z_{L} \text {, } \\
& I_{79} \equiv B_{K} C_{K L} C_{L M} Z_{M}, \quad I_{80} \equiv B_{K} \dot{C}_{K L} Z_{L}, \\
& I_{81} \equiv B_{K} \dot{C}_{K L} \dot{C}_{L M} Z_{M}, \quad I_{82} \equiv B_{K} H_{K L} Z_{L} \text {, } \\
& I_{83} \equiv B_{K} \dot{H}_{K L} Z_{L}, \quad I_{84} \equiv B_{K} C_{K L} \dot{C}_{L M} Z_{M}, \\
& I_{85} \equiv B_{K} C_{K L} H_{L M} Z_{M}, \quad I_{86} \equiv B_{K} C_{K L} \dot{H}_{L M} Z_{M} \text {, } \\
& I_{87} \equiv B_{K} \dot{C}_{K L} H_{L M} Z_{M}, \quad I_{88} \equiv B_{K} \dot{C}_{K L} \dot{H}_{L M} Z_{M}, \\
& I_{89} \equiv B_{K} H_{K L} \dot{H}_{L M} Z_{M} \text {, } \\
& I_{90} \equiv B_{K} C_{K L} \dot{C}_{L M} H_{M N} Z_{N} \text {, } \\
& I_{91} \equiv B_{K} C_{K L} \dot{C}_{L M} \dot{H}_{M N} Z_{N} \text {, } \\
& I_{92} \equiv B_{K} C_{K L} H_{L M} \dot{H}_{M N} Z_{N} \text {, } \\
& I_{93} \equiv B_{K} \dot{C}_{K L} H_{L M} \dot{H}_{M N} Z_{N} \text {, } \\
& I_{94} \equiv B_{K} C_{K L} C_{L M} \dot{C}_{M N} H_{N S} Z_{S} \text {, } \\
& I_{95} \equiv B_{K} \dot{C}_{K L} \dot{C}_{L M} H_{M N} C_{N S} B_{S} \text {, } \\
& I_{96} \equiv B_{K} C_{K L} C_{L M} \dot{C}_{M N} \dot{H}_{N S} Z_{S}, \\
& I_{97} \equiv B_{K} \dot{C}_{K L} \dot{C}_{L M} H_{M N} \dot{H}_{N S} Z_{S} \text {, } \\
& I_{98} \equiv B_{K} C_{K L} \dot{C}_{L M} H_{M N} \dot{H}_{N S} Z_{S} .
\end{aligned}
$$

However, the main function that needs to be obtained is in the form of arguments of the scalar isotropic function $\Re$, that is, $C_{K L}, \dot{C}_{K L}, H_{K L}, \dot{H}_{K L}, B_{K}, Z_{K}, V_{K}$. Because the scalar 
$\Re$ is a bilinear function of the vector $\mathbf{V}$, it depends on the following invariants in addition to those provided in (44):

$$
\begin{aligned}
& K_{1} \equiv V_{K} B_{K}, \quad K_{2} \equiv V_{K} Z_{K}, \\
& K_{3} \equiv V_{K} C_{K L} B_{L}, \quad K_{4} \equiv V_{K} C_{K L} Z_{L} \text {, } \\
& K_{5} \equiv V_{K} \dot{C}_{K L} B_{L}, \quad K_{6} \equiv V_{K} \dot{C}_{K L} Z_{L}, \\
& K_{7} \equiv V_{K} H_{K L} B_{L}, \quad K_{8} \equiv V_{K} H_{K L} Z_{L} \text {, } \\
& K_{9} \equiv V_{K} \dot{H}_{K L} B_{L}, \quad K_{10} \equiv V_{K} \dot{H}_{K L} Z_{L}, \\
& K_{11} \equiv V_{K} C_{K M} C_{M L} B_{L}, \quad K_{12} \equiv V_{K} C_{K M} C_{M L} Z_{L} \text {, } \\
& K_{13} \equiv V_{K} \dot{C}_{K M} \dot{C}_{M L} B_{L}, \quad K_{14} \equiv V_{K} \dot{C}_{K M} \dot{C}_{M L} Z_{L} \text {, } \\
& K_{15} \equiv V_{K} C_{K L} \dot{C}_{L M} B_{M}, \quad K_{16} \equiv V_{K} C_{K L} \dot{C}_{L M} Z_{M} \text {, } \\
& K_{17} \equiv V_{K} C_{K L} H_{L M} B_{M}, \quad K_{18} \equiv V_{K} C_{K L} H_{L M} Z_{M} \text {, } \\
& K_{19} \equiv V_{K} C_{K L} \dot{H}_{L M} B_{M}, \quad K_{20} \equiv V_{K} C_{K L} \dot{H}_{L M} Z_{M} \text {, } \\
& K_{21} \equiv V_{K} \dot{C}_{K L} H_{L M} B_{M} \text {, } \\
& K_{22} \equiv V_{K} \dot{C}_{K L} H_{L M} Z_{M} \text {, } \\
& K_{23} \equiv V_{K} \dot{C}_{K L} \dot{H}_{L M} B_{M} \text {, } \\
& K_{24} \equiv V_{K} \dot{C}_{K L} \dot{H}_{L M} Z_{M} \text {, } \\
& K_{25} \equiv V_{K} H_{K L} \dot{H}_{L M} B_{M} \text {, } \\
& K_{26} \equiv V_{K} H_{K L} \dot{H}_{L M} Z_{M} \text {, } \\
& K_{27} \equiv V_{K} C_{K L} \dot{C}_{L M} H_{M N} B_{N} \text {, } \\
& K_{28} \equiv V_{K} C_{K L} \dot{C}_{L M} H_{M N} Z_{N} \text {, } \\
& K_{29} \equiv V_{K} C_{K L} H_{L M} \dot{H}_{M N} B_{N} \text {, } \\
& K_{30} \equiv V_{K} C_{K L} H_{L M} \dot{H}_{M N} Z_{N} \text {, } \\
& K_{31} \equiv V_{K} \dot{C}_{K L} H_{L M} \dot{H}_{M N} B_{N} \text {, } \\
& K_{32} \equiv V_{K} \dot{C}_{K L} H_{L M} \dot{H}_{M N} Z_{N} \text {, } \\
& K_{33} \equiv V_{K} C_{K L} C_{L M} \dot{C}_{M N} H_{N S} B_{S} \text {, } \\
& K_{34} \equiv V_{K} C_{K L} C_{L M} \dot{C}_{M N} H_{N S} Z_{S} \text {, } \\
& K_{35} \equiv V_{K} C_{K L} C_{L M} \dot{C}_{M N} \dot{H}_{N S} B_{S} \text {, } \\
& K_{36} \equiv V_{K} C_{K L} C_{L M} \dot{C}_{M N} \dot{H}_{N S} Z_{S} \text {, } \\
& K_{37} \equiv V_{K} \dot{C}_{K L} \dot{C}_{L M} H_{M N} C_{N S} B_{S}, \\
& K_{38} \equiv V_{K} \dot{C}_{K L} \dot{C}_{L M} H_{M N} C_{N S} Z_{S} \text {, } \\
& K_{39} \equiv V_{K} \dot{C}_{K L} \dot{C}_{L M} H_{M N} \dot{H}_{N S} B_{S} \text {, } \\
& K_{40} \equiv V_{K} \dot{C}_{K L} \dot{C}_{L M} H_{M N} \dot{H}_{N S} Z_{S} \text {, } \\
& K_{41} \equiv V_{K} C_{K L} \dot{C}_{L M} H_{M N} \dot{H}_{N S} B_{S} \text {, }
\end{aligned}
$$

$$
\begin{gathered}
K_{42} \equiv V_{K} C_{K L} \dot{C}_{L M} H_{M N} \dot{H}_{N S} Z_{S}, \\
K_{43} \equiv V_{K} V_{K}, \quad K_{44} \equiv V_{K} C_{K L} V_{L}, \\
K_{45} \equiv V_{K} \dot{C}_{K L} V_{L}, \quad K_{46} \equiv V_{K} H_{K L} V_{L}, \\
K_{47} \equiv V_{K} \dot{H}_{K L} V_{L}, \quad K_{48} \equiv V_{K} C_{K L} C_{L M} V_{M}, \\
K_{49} \equiv V_{K} \dot{C}_{K L} \dot{C}_{L M} V_{M}, \quad K_{50} \equiv V_{K} C_{K L} \dot{C}_{L M} V_{M}, \\
K_{51} \equiv V_{K} C_{K L} H_{L M} V_{M}, \quad K_{52} \equiv V_{K} C_{K L} \dot{H}_{L M} V_{M}, \\
K_{53} \equiv V_{K} \dot{C}_{K L} H_{L M} V_{M}, \\
K_{54} \equiv V_{K} \dot{C}_{K L} \dot{H}_{L M} V_{M}, \\
K_{55} \equiv V_{K} H_{K L} \dot{H}_{L M} V_{M}, \\
K_{56} \equiv V_{K} C_{K L} \dot{C}_{L M} H_{M N} V_{N}, \\
K_{57} \equiv V_{K} C_{K L} \dot{C}_{L M} \dot{H}_{M N} V_{N}, \\
K_{58} \equiv V_{K} C_{K L} H_{L M} \dot{H}_{M N} V_{N}, \\
K_{59} \equiv V_{K} \dot{C}_{K L} H_{L M} \dot{H}_{M N} V_{N}, \\
K_{60} \equiv V_{K} C_{K L} C_{L M} \dot{C}_{M N} H_{N S} V_{S}, \\
K_{61} \equiv V_{K} C_{K L} C_{L M} \dot{C}_{M N} \dot{H}_{N S} V_{S}, \\
K_{62} \equiv V_{K} \dot{C}_{K L} \dot{C}_{L M} H_{M N} C_{N S} V_{S}, \\
K_{63} \equiv V_{K} \dot{C}_{K L} \dot{C}_{L M} H_{M N} \dot{H}_{N S} V_{S}, \\
K_{64} \equiv V_{K} C_{K L} \dot{C}_{L M} H_{M N} \dot{H}_{N S} V_{S} .
\end{gathered}
$$

In this situation, the scalar function $\Re$ defined by expression (40) must be bilinear in terms of invariants $K_{i}(i=1,2$, $\ldots, 42)$ in expression (45) and linear in terms of invariants $K_{i}(i=43,44, \ldots, 64)$. In this case the following expression can be recorded for function $\Re$ :

$$
\begin{aligned}
\Re(\underline{\underline{C}}, \underline{\underline{C}}, \underline{A}, \underline{B}, \underline{V}) \\
=\sum_{\alpha=1}^{42} \sum_{\beta=1}^{42} \lambda_{\alpha \beta} K_{\alpha} K_{\beta}+\lambda_{0} K_{43} \\
\quad+\lambda_{1} K_{44}+\cdots+\lambda_{m} K_{n} \\
m=2,3,4, \ldots, 20 ; \quad n=45,46,47, \ldots, 64 .
\end{aligned}
$$

Coefficients $\lambda_{0}, \lambda_{1}, \ldots, \lambda_{m}, m=2,3,4, \ldots, 20$ and $\lambda_{\alpha \beta}(\alpha, \beta=1,2,3, \ldots, 42)$ in (46) are each a scalar function of the invariants provided in (44). Besides, symmetry condition $\lambda_{\alpha \beta}=\lambda_{\beta \alpha}$ is true for the coefficients $\lambda_{\alpha \beta}$. Using relationship (42), due to the assumptions made in this study concerning interactions, terms of tensors $\mathbf{C}, \dot{\mathbf{C}}, \mathbf{H}$, and $\dot{\mathbf{H}}$ have been only considered on the first grade of the external product of fiber 
vectors $\mathbf{B}$ and $\mathbf{Z}$, only even number components have been considered, yielding the dissipative stress as follows:

$$
\begin{aligned}
& { }_{D} T_{P R}=\lambda_{0} \delta_{P R}+\lambda_{1} C_{P R}+\lambda_{2} \dot{C}_{P R}+\lambda_{3} H_{P R} \\
& +\lambda_{4} \dot{H}_{P R}+\lambda_{11} B_{P} B_{R} \\
& +\lambda_{12}\left(B_{P} Z_{R}+Z_{P} B_{R}\right) \\
& +\lambda_{13}\left(B_{P} B_{L} C_{L R}+C_{P K} B_{K} B_{R}\right) \\
& +\lambda_{14}\left(B_{P} Z_{L} C_{L R}+C_{P K} Z_{K} B_{R}\right) \\
& +\lambda_{15}\left(B_{P} B_{L} \dot{C}_{L R}+\dot{C}_{P K} B_{K} B_{R}\right) \\
& +\lambda_{16}\left(B_{P} Z_{L} \dot{C}_{L R}+\dot{C}_{P K} Z_{K} B_{R}\right) \\
& +\lambda_{17}\left(B_{P} B_{L} H_{L R}+H_{P K} B_{K} B_{R}\right) \\
& +\lambda_{18}\left(B_{P} Z_{L} H_{L R}+H_{P K} Z_{K} B_{R}\right) \\
& +\lambda_{19}\left(B_{P} B_{L} \dot{H}_{L R}+\dot{H}_{P K} B_{K} B_{R}\right) \\
& +\lambda_{1,10}\left(B_{P} Z_{L} \dot{H}_{L R}+\dot{H}_{P K} Z_{K} B_{R}\right) \\
& +\lambda_{22} Z_{P} Z_{R}+\lambda_{23}\left(Z_{P} B_{L} C_{L R}+C_{P K} B_{K} Z_{R}\right) \\
& +\lambda_{24}\left(Z_{P} Z_{L} C_{L R}+C_{P K} Z_{K} Z_{R}\right) \\
& +\lambda_{25}\left(Z_{P} B_{L} \dot{C}_{L R}+\dot{C}_{P K} B_{K} Z_{R}\right) \\
& +\lambda_{26}\left(Z_{P} Z_{L} \dot{C}_{L R}+\dot{C}_{P K} Z_{K} Z_{R}\right) \\
& +\lambda_{27}\left(Z_{P} B_{L} H_{L R}+H_{P K} B_{K} Z_{R}\right) \\
& +\lambda_{28}\left(Z_{P} Z_{L} H_{L R}+H_{P K} Z_{K} Z_{R}\right) \\
& +\lambda_{29}\left(Z_{P} B_{L} \dot{H}_{L R}+\dot{H}_{P K} B_{K} Z_{R}\right) \\
& +\lambda_{2,10}\left(Z_{P} Z_{L} \dot{H}_{L R}+\dot{H}_{P K} Z_{K} Z_{R}\right) \text {. }
\end{aligned}
$$

Because mechanical interactions and effect of damage have been assumed to be linear, coefficients in (47) are each a scalar function of invariants that do not contain square or higher grade terms of tensors $\mathbf{C}$ and $\dot{\mathbf{C}}$ or terms in the forms

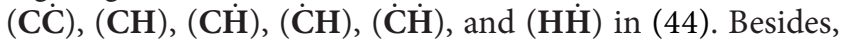
taking into account that values of the invariants $I_{36}$ and $I_{57}$ are equal to 1 due to the inextensibility of fiber families and values of the invariants $I_{33}$ and $I_{34}$ are equal to 1 because they are unit vectors pertaining to the distribution of fibers $\mathbf{B}$ and $\mathbf{Z}$ before deformation, invariants on which the said coefficients depend have been recorded as follows in terms of $\left(E_{K L}\right)$ and $\left(\dot{E}_{K L}\right)$.

$$
\begin{gathered}
J_{1}=3+2 E_{K L}, \quad J_{2}=2 \dot{E}_{K K}, \\
J_{3}=H_{K K}, \quad J_{4}=\dot{H}_{K K}, \\
J_{5}=B_{K} Z_{K}, \quad J_{6}=2 B_{K} \dot{E}_{K L} B_{L}, \\
J_{7}=B_{K} H_{K L} B_{L}, \quad J_{8}=B_{K} \dot{H}_{K L} B_{L},
\end{gathered}
$$

$$
\begin{gathered}
J_{9}=2 Z_{K} \dot{E}_{K L} Z_{L}, \quad J_{10}=Z_{K} H_{K L} Z_{L}, \\
J_{11}=Z_{K} \dot{H}_{K L} Z_{L}, \quad J_{12}=B_{K} Z_{K}+2 B_{K} E_{K L} B_{L}, \\
J_{13}=2 B_{K} \dot{E}_{K L} Z_{L}, \quad J_{14}=B_{K} H_{K L} Z_{L}, \\
J_{15}=B_{K} \dot{H}_{K L} Z_{L} .
\end{gathered}
$$

Coefficients in (47) can be recorded as a scalar function of the invariants in (48) can be expressed as follows.

$$
\lambda_{\alpha}=\beta_{0}+\sum_{i=1}^{15} \beta_{i} J_{i}, \quad 0 \leq \alpha \leq 2,10
$$

Here the following definitions have been used.

$(\alpha=0 \Rightarrow \beta=a, \alpha=1 \Rightarrow \beta=b, \alpha=2 \Rightarrow \beta=$ $c, \alpha=3 \Rightarrow \beta=d, \alpha=4 \Rightarrow \beta=f, \alpha=11 \Rightarrow \beta=g$, $\alpha=12 \Rightarrow \beta=h, \alpha=13 \Rightarrow \beta=i, \alpha=14 \Rightarrow \beta=k, \alpha=$ $15 \Rightarrow \beta=l, \alpha=16 \Rightarrow \beta=m, \alpha=17 \Rightarrow \beta=n, \alpha=18 \Rightarrow$ $\beta=p, \alpha=19 \Rightarrow \beta=r, \alpha=1,10 \Rightarrow \beta=q, \alpha=22 \Rightarrow \beta=$ $o, \alpha=23 \Rightarrow \beta=s, \alpha=24 \Rightarrow \beta=s, \alpha=25 \Rightarrow \beta=t, \alpha=$ $26 \Rightarrow \beta=u, \alpha=27 \Rightarrow \beta=w, \alpha=28 \Rightarrow \beta=v, \alpha=29 \Rightarrow$ $\beta=y, \alpha=2,10 \Rightarrow \beta=z)$.

Using expressions (49) in (47), expressing the tensors $C_{K L}$ and $\dot{C}_{K L}$ in terms of the tensors $E_{K L}$ and $\dot{E}_{K L}$, taking mechanical interactions, effect of damage interactions, and assumptions made concerning the fibers into account, the following has been expressed:

$$
\begin{aligned}
{ }_{D} T_{P R}= & \Gamma_{1} \delta_{P R}+\Gamma_{2} E_{K K} \delta_{P R}+\Gamma_{3} \dot{E}_{K K} \delta_{P R} \\
& +\Gamma_{4} H_{K K} \delta_{P R}+\Gamma_{5} \dot{H}_{K K} \delta_{P R}+\Gamma_{6} B_{K} \dot{E}_{K L} B_{L} \delta_{P R} \\
& +\Gamma_{7} B_{K} H_{K L} B_{L} \delta_{P R}+\Gamma_{8} B_{K} \dot{H}_{K L} B_{L} \delta_{P R} \\
& +\Gamma_{9} Z_{K} \dot{E}_{K L} Z_{L} \delta_{P R}+\Gamma_{10} Z_{K} H_{K L} Z_{L} \delta_{P R} \\
& +\Gamma_{11} Z_{K} \dot{H}_{K L} Z_{L} \delta_{P R}+\Gamma_{12} E_{P R} \\
& +\Gamma_{13} \dot{E}_{P R}+\Gamma_{14} H_{P R}+\Gamma_{15} \dot{H}_{P R} \\
& +\Gamma_{16} B_{P} B_{R}+\Gamma_{17} E_{K K} B_{P} B_{R} \\
& +\Gamma_{18} \dot{E}_{K K} B_{P} B_{R}+\Gamma_{19} H_{K K} B_{P} B_{R} \\
& +\Gamma_{20} \dot{H}_{K K} B_{P} B_{R}+\Gamma_{21} Z_{K} \dot{E}_{K L} Z_{L} B_{P} B_{R} \\
& +\Gamma_{22} Z_{K} H_{K L} Z_{L} B_{P} B_{R}+\Gamma_{23} Z_{K} \dot{H}_{K L} Z_{L} B_{P} B_{R} \\
& +\Gamma_{24} B_{K} Z_{K}\left(B_{P} Z_{R}+Z_{P} B_{R}\right) \\
& +\Gamma_{25} B_{K} E_{K L} Z_{L}\left(B_{P} Z_{R}+Z_{P} B_{R}\right) \\
& +\Gamma_{26} B_{K} \dot{E}_{K L} Z_{L}\left(B_{P} Z_{R}+Z_{P} B_{R}\right) \\
& +\Gamma_{27} B_{K} H_{K L} Z_{L}\left(B_{P} Z_{R}+Z_{P} B_{R}\right) \\
& +\Gamma_{28} B_{K} \dot{H}_{K L} Z_{L}\left(B_{P} Z_{R}+Z_{P} B_{R}\right) \\
& +\Gamma_{29}\left(B_{P} B_{L} E_{L P}+E_{P L} B_{L} B_{R}\right) \\
&
\end{aligned}
$$




$$
\begin{aligned}
& +\Gamma_{30} B_{K} Z_{K}\left(B_{P} Z_{L} E_{L P}+E_{P L} Z_{L} B_{R}\right) \\
& +\Gamma_{31}\left(B_{P} B_{L} \dot{E}_{L P}+\dot{E}_{P L} B_{L} B_{R}\right) \\
& +\Gamma_{32} B_{K} Z_{K}\left(B_{P} Z_{L} \dot{E}_{L P}+\dot{E}_{P L} Z_{L} B_{R}\right) \\
& +\Gamma_{33}\left(B_{P} B_{L} H_{L P}+H_{P L} B_{L} B_{R}\right) \\
& +\Gamma_{34} B_{K} Z_{K}\left(B_{P} Z_{L} H_{L P}+H_{P L} Z_{L} B_{R}\right) \\
& +\Gamma_{35}\left(B_{P} B_{L} \dot{H}_{L P}+\dot{H}_{P L} B_{L} B_{R}\right) \\
& +\Gamma_{36} B_{K} Z_{K}\left(B_{P} Z_{L} \dot{H}_{L P}+\dot{H}_{P L} Z_{L} B_{R}\right) \\
& +\Gamma_{37} Z_{P} Z_{R}+\Gamma_{38} E_{K K} Z_{P} Z_{R} \\
& +\Gamma_{39} \dot{E}_{K K} Z_{P} Z_{R}+\Gamma_{40} H_{K K} Z_{P} Z_{R} \\
& +\Gamma_{41} \dot{H}_{K K} Z_{P} Z_{R}+\Gamma_{42} B_{K} \dot{E}_{K L} B_{L} Z_{P} Z_{R} \\
& +\Gamma_{43} B_{K} H_{K L} B_{L} Z_{P} Z_{R}+\Gamma_{44} B_{K} \dot{H}_{K L} B_{L} Z_{P} Z_{R} \\
& +\Gamma_{45} B_{K} Z_{K}\left(Z_{P} B_{L} E_{L P}+E_{P L} B_{L} Z_{R}\right) \\
& +\Gamma_{52}\left(Z_{P} Z_{L} \dot{H}_{L P}+\dot{H}_{P L} Z_{L} Z_{R}\right) \\
& +\Gamma_{46}\left(Z_{P} Z_{L} E_{L P}+E_{P L} Z_{L} Z_{R}\right) \\
& +\Gamma_{50} B_{K} Z_{K}\left(Z_{P} B_{L} \dot{E}_{L P}+\dot{E}_{P L} B_{L} Z_{R}\right) \\
& +\Gamma_{48}\left(Z_{P} Z_{L} \dot{E}_{L P}+\dot{E}_{P L} Z_{L} Z_{R}\right) \\
& +\Gamma_{49} B_{K} Z_{K}\left(Z_{P} B_{L} H_{L P}+H_{P L} B_{L} Z_{R}\right) \\
& \left.+H_{P L} Z_{L} Z_{R}\right) \\
& \left.+\Gamma_{P} B_{L} \dot{H}_{L P}+\dot{H}_{P L} B_{L} Z_{R}\right) \\
& \left.+\Gamma_{5}\right)
\end{aligned}
$$

Coefficients $\Gamma_{k}(k=1,2,3, \ldots, 52)$ in (50) have been depending on the medium temperature $\theta$ and $\lambda_{\alpha \beta}$.

Because the tensors $C_{K L}$ and $\dot{C}_{K L}$ can be expressed in terms of the tensors $E_{K L}$ and $\dot{E}_{K L}$, expression (21) imposes a constraint on the coefficients in (50). Accordingly, the following expression can be recorded:

$$
\begin{aligned}
0= & \Gamma_{1} \delta_{P R}+\Gamma_{2} E_{K K} \delta_{P R}+\Gamma_{4} H_{K K} \delta_{P R} \\
& +\Gamma_{7} B_{K} H_{K L} B_{L} \delta_{P R}+\Gamma_{10} Z_{K} H_{K L} Z_{L} \delta_{P R}+\Gamma_{12} E_{P R} \\
& +\Gamma_{14} H_{P R}+\Gamma_{16} B_{P} B_{R}+\Gamma_{17} E_{K K} B_{P} B_{R} \\
& +\Gamma_{19} H_{K K} B_{P} B_{R}+\Gamma_{22} Z_{K} H_{K L} Z_{L} B_{P} B_{R} \\
& +\Gamma_{24} B_{K} Z_{K}\left(B_{P} Z_{R}+Z_{P} B_{R}\right) \\
& +\Gamma_{25} B_{K} E_{K L} Z_{L}\left(B_{P} Z_{R}+Z_{P} B_{R}\right) \\
& +\Gamma_{27} B_{K} H_{K L} Z_{L}\left(B_{P} Z_{R}+Z_{P} B_{R}\right) \\
& +\Gamma_{29}\left(B_{P} B_{L} E_{L P}+E_{P L} B_{L} B_{R}\right)
\end{aligned}
$$

$$
\begin{aligned}
& +\Gamma_{30} B_{K} Z_{K}\left(B_{P} Z_{L} E_{L P}+E_{P L} Z_{L} B_{R}\right) \\
& +\Gamma_{33}\left(B_{P} B_{L} H_{L P}+H_{P L} B_{L} B_{R}\right) \\
& +\Gamma_{34} B_{K} Z_{K}\left(B_{P} Z_{L} H_{L P}+H_{P L} Z_{L} B_{R}\right) \\
& +\Gamma_{37} Z_{P} Z_{R}+\Gamma_{38} E_{K K} Z_{P} Z_{R} \\
& +\Gamma_{40} H_{K K} Z_{P} Z_{R}+\Gamma_{43} B_{K} H_{K L} B_{L} Z_{P} Z_{R} \\
& +\Gamma_{45} B_{K} Z_{K}\left(Z_{P} B_{L} E_{L P}+E_{P L} B_{L} Z_{R}\right) \\
& +\Gamma_{46}\left(Z_{P} Z_{L} E_{L P}+E_{P L} Z_{L} Z_{R}\right) \\
& +\Gamma_{49} B_{K} Z_{K}\left(Z_{P} B_{L} H_{L P}+H_{P L} B_{L} Z_{R}\right) \\
& +\Gamma_{50}\left(Z_{P} Z_{L} H_{L P}+H_{P L} Z_{L} Z_{R}\right) .
\end{aligned}
$$

Because, according to expression (51), the above-mentioned are arbitrary, the necessary and sufficient condition for validity of this equation is $\Gamma_{y}=0$ coefficients being zero ( $y=$ $1,2,4,7,10,12,14,16,17,19,22,24,25,27,29,30,33,34,37$, $38,40,43,45,46,49,50)$. In this situation, expression providing the dissipative stress is obtained as follows:

$$
\begin{aligned}
{ }_{D} T_{P R}= & \Gamma_{3} \dot{E}_{K K} \delta_{P R}+\Gamma_{5} \dot{H}_{K K} \delta_{P R}+\Gamma_{6} B_{K} \dot{E}_{K L} B_{L} \delta_{P R} \\
& +\Gamma_{8} B_{K} \dot{H}_{K L} B_{L} \delta_{P R}+\Gamma_{9} Z_{K} \dot{E}_{K L} Z_{L} \delta_{P R} \\
& +\Gamma_{11} Z_{K} \dot{H}_{K L} Z_{L} \delta_{P R}+\Gamma_{13} \dot{E}_{P R} \\
& +\Gamma_{15} \dot{H}_{P R}+\Gamma_{18} \dot{E}_{K K} B_{P} B_{R}+\Gamma_{20} \dot{H}_{K K} B_{P} B_{R} \\
& +\Gamma_{21} Z_{K} \dot{E}_{K L} Z_{L} B_{P} B_{R}+\Gamma_{23} Z_{K} \dot{H}_{K L} Z_{L} B_{P} B_{R} \\
& +\Gamma_{26} B_{K} \dot{E}_{K L} Z_{L}\left(B_{P} Z_{R}+Z_{P} B_{R}\right) \\
& +\Gamma_{28} B_{K} \dot{H}_{K L} Z_{L}\left(B_{P} Z_{R}+Z_{P} B_{R}\right) \\
& +\Gamma_{31}\left(B_{P} B_{L} \dot{E}_{L P}+\dot{E}_{P L} B_{L} B_{R}\right) \\
& +\Gamma_{32} B_{K} Z_{K}\left(B_{P} Z_{L} \dot{E}_{L P}+\dot{E}_{P L} Z_{L} B_{R}\right) \\
& +\Gamma_{35}\left(B_{P} B_{L} \dot{H}_{L P}+\dot{H}_{P L} B_{L} B_{R}\right) \\
& +\Gamma_{36} B_{K} Z_{K}\left(B_{P} Z_{L} \dot{H}_{L P}+\dot{H}_{P L} Z_{L} B_{R}\right) \\
& +\Gamma_{39} \dot{E}_{K K} Z_{P} Z_{R}+\Gamma_{41} \dot{H}_{K K} Z_{P} Z_{R} \\
& +\Gamma_{51} B_{K} Z_{K}\left(Z_{P} B_{L} \dot{H}_{L P}+\dot{H}_{P L} B_{L} Z_{R}\right) \\
& \left.+\Gamma_{42} B_{K} \dot{E}_{K L} B_{L} Z_{P} Z_{R}+\Gamma_{44} B_{K} \dot{H}_{K L} B_{L} Z_{K} Z_{P} Z_{R} \dot{H}_{P L} Z_{L} Z_{R}\right) \\
& \left.+\Gamma_{P} \dot{E}_{L P}+\dot{E}_{P L} B_{L} Z_{R}\right) \\
& \left.\dot{E}_{L P}+\dot{E}_{P L} Z_{L} Z_{R}\right) \\
& \left.+\Gamma_{2}\right) \\
&
\end{aligned}
$$

Equations of the elastic stress provided by expression (37) and of the dissipative stress provided by expression (52) are 
substituted into (22); thus the total stress has been obtained as follows:

$$
\begin{aligned}
& T_{P R}=-p C_{P R}^{-1}+\Gamma_{b} B_{P} B_{R}+\Gamma_{z} Z_{P} Z_{R}+\alpha_{2} E_{K K} \delta_{P R} \\
& +\alpha_{3} H_{K K} \delta_{P R}+\alpha_{4} B_{K} H_{K N} B_{N} \delta_{P R} \\
& +\alpha_{5} Z_{K} H_{K N} Z_{N} \delta_{P R}+\alpha_{6} E_{P R}+\alpha_{7} E_{K K} B_{P} B_{R} \\
& +\alpha_{8} H_{K K} B_{P} B_{R}+\alpha_{9} Z_{K} H_{K N} Z_{N} B_{P} B_{R} \\
& +\alpha_{10}\left[B_{P} B_{R}+\left(B_{P} B_{L} E_{L R}+E_{P L} B_{L} B_{R}\right)\right] \\
& +\alpha_{11} E_{K K} Z_{P} Z_{R}+\alpha_{12} H_{K K} Z_{P} Z_{R} \\
& +\alpha_{13} B_{K} H_{K N} B_{N} Z_{P} Z_{R} \\
& +\alpha_{14}\left[Z_{P} Z_{R}+\left(Z_{P} Z_{L} E_{L R}+E_{P L} Z_{L} Z_{R}\right)\right] \\
& +\alpha_{15} B_{K} Z_{K} B_{P} Z_{R}+\alpha_{16} B_{K} E_{K N} Z_{N} B_{P} Z_{R} \\
& +\alpha_{17} B_{K} H_{K N} Z_{N} B_{P} Z_{R} \\
& +\alpha_{18} B_{K} Z_{K}\left(B_{P} Z_{L} E_{L R}+E_{P L} B_{L} Z_{R}\right) \\
& +\alpha_{19}\left(B_{P} B_{L} H_{L R}+H_{P L} B_{L} B_{R}\right) \\
& +\alpha_{20}\left(Z_{P} Z_{L} H_{L R}+H_{P L} Z_{L} Z_{R}\right) \\
& +\alpha_{21} B_{K} Z_{K}\left(B_{P} Z_{L} H_{L R}+H_{P L} B_{L} Z_{R}\right) \\
& +\alpha_{22} H_{P R}+\Gamma_{3} \dot{E}_{K K} \delta_{P R}+\Gamma_{5} \dot{H}_{K K} \delta_{P R} \\
& +\Gamma_{6} B_{K} \dot{E}_{K L} B_{L} \delta_{P R}+\Gamma_{8} B_{K} \dot{H}_{K L} B_{L} \delta_{P R} \\
& +\Gamma_{9} Z_{K} \dot{E}_{K L} Z_{L} \delta_{P R}+\Gamma_{11} Z_{K} \dot{H}_{K L} Z_{L} \delta_{P R} \\
& +\Gamma_{13} \dot{E}_{P R}+\Gamma_{15} \dot{H}_{P R}+\Gamma_{18} \dot{E}_{K K} B_{P} B_{R} \\
& +\Gamma_{20} \dot{H}_{K K} B_{P} B_{R}+\Gamma_{21} Z_{K} \dot{E}_{K L} Z_{L} B_{P} B_{R} \\
& +\Gamma_{23} Z_{K} \dot{H}_{K L} Z_{L} B_{P} B_{R} \\
& +\Gamma_{26} B_{K} \dot{E}_{K L} Z_{L}\left(B_{P} Z_{R}+Z_{P} B_{R}\right) \\
& +\Gamma_{28} B_{K} \dot{H}_{K L} Z_{L}\left(B_{P} Z_{R}+Z_{P} B_{R}\right) \\
& +\Gamma_{31}\left(B_{P} B_{L} \dot{E}_{L P}+\dot{E}_{P L} B_{L} B_{R}\right) \\
& +\Gamma_{32} B_{K} Z_{K}\left(B_{P} Z_{L} \dot{E}_{L P}+\dot{E}_{P L} Z_{L} B_{R}\right) \\
& +\Gamma_{35}\left(B_{P} B_{L} \dot{H}_{L P}+\dot{H}_{P L} B_{L} B_{R}\right) \\
& +\Gamma_{36} B_{K} Z_{K}\left(B_{P} Z_{L} \dot{H}_{L P}+\dot{H}_{P L} Z_{L} B_{R}\right) \\
& +\Gamma_{39} \dot{E}_{K K} Z_{P} Z_{R}+\Gamma_{41} \dot{H}_{K K} Z_{P} Z_{R} \\
& +\Gamma_{42} B_{K} \dot{E}_{K L} B_{L} Z_{P} Z_{R}+\Gamma_{44} B_{K} \dot{H}_{K L} B_{L} Z_{P} Z_{R} \\
& +\Gamma_{47} B_{K} Z_{K}\left(Z_{P} B_{L} \dot{E}_{L P}+\dot{E}_{P L} B_{L} Z_{R}\right) \\
& +\Gamma_{48}\left(Z_{P} Z_{L} \dot{E}_{L P}+\dot{E}_{P L} Z_{L} Z_{R}\right)
\end{aligned}
$$

$$
\begin{aligned}
& +\Gamma_{51} B_{K} Z_{K}\left(Z_{P} B_{L} \dot{H}_{L P}+\dot{H}_{P L} B_{L} Z_{R}\right) \\
& +\Gamma_{52}\left(Z_{P} Z_{L} \dot{H}_{L P}+\dot{H}_{P L} Z_{L} Z_{R}\right) .
\end{aligned}
$$

Kelvin-Voigt body composing a viscoelastic model is defined as a continuum model where stress depends on the rate of deformation together with the deformation. The stress constitutive equation of the linear Kelvin-Voigt body has been expressed as $t_{k l}=\Sigma_{k l m n}(\theta, \mathbf{x}) \epsilon_{m n}+\Gamma_{k l m n}(\theta, \mathbf{x}) \dot{\epsilon}_{m n}$. $\Sigma_{k l m n}$ coefficient bears the symmetry characteristics given as $\Sigma_{k l m n}=\Sigma_{l k m n}=\Sigma_{k l n m}=\Sigma_{m n k l}$, whereas $\Gamma_{k l m n}$ coefficient bears the symmetry characteristics given as $\Gamma_{k l m n}=\Gamma_{l k m n}=$ $\Gamma_{k l n m}$. This symmetry characteristic decreases the number of components of material module $\Sigma_{k l m n}$ to 21 and the number of components of material module $\Gamma_{k l m n}$ to 36 . Besides, as a result of Onsager principle in the linear reversible thermodynamics, it has been assumed that relaxation tensor $\Gamma_{k l m n}$ has a symmetry characteristic as $\Gamma_{k l m n}=\Gamma_{m n k l}$. According to this symmetry condition, the number of components pertaining to material module $\Gamma_{k l m n}$ is thus reduced to 21 .

\section{Concluding Remarks}

This paper presents a continuum damage model based on fundamental concepts of continuum mechanics for the linear viscoelastic behavior of incompressible composites with microcracks that consist of an isotropic matrix reinforced by inextensible two families of fibers having an arbitrary distribution. "Damage" is expressed by two symmetric, second rank tensors which are related to the total areas of "active" and "passive" microcracks within a representative volume element of the multifractured material. The matrix material has been assumed to be an isotropic medium; however, due to the distributions of fibers and the existence of microcracks, it has gained the property of a directed object, thus gaining the appearance of an anisotropic structure. Inextensibility of the fibers is an assumption which is widely used in practice. Thus, fiber families are assumed to be inextensible and composite medium is assumed to be incompressible. In this context, the composite expresses itself behaviorally in terms of the elastic stress, the dissipative stress, and the strain energy density release rate. To obtain a more concrete expression of nonlinear constitutive equations of the elastic stress and the strain energy density release rate given by expressions (33) and (34), derivatives of $\Sigma$ must be known according to the arguments it depends on. Thus, stress potential $\Sigma$ has been represented by a second degree polynomial and its derivatives according to its invariants have been calculated. In this study, mechanical interactions and effect of damage have been assumed to be linear. Furthermore, since the matrix material has to remain insensitive to directional changes along fibers, even-numbered exterior products of vector fields representing fiber distributions have been considered. The linear constitutive equations of the elastic stress and the strain energy density release rate have been given by expressions (37) and (38). From these equations, it can be seen that the deformation field, the damage tensor, distribution of fibers, 
and interactions of them all contribute to the creation of elastic stress and strain energy density release rate.

Equation (47) obtained by using the invariants of the arguments depends on the dissipative stress, since the dissipative stress is a vector-valued isotropic function. Coefficients in this equation have been expressed in terms of the invariants on which they depend and each term has been calculated. When these calculations are made, the mechanical interaction and the effect of damage have been assumed to be linear and even number vector components of fiber vectors have been included in the operations, since the composite remains indifferent to changes of direction along the fibers. The linear constitutive equation of the dissipative stress has been expressed by (52). As understood from this equation, separate and common interactions of the fiber distribution fields with the rate of deformation and the change in time of the damage tensor contribute to the formation of dissipative stress. Substituting (37) and (52) into the expression (22), the total stress has been obtained in (53).

This paper is concerned with developing the continuum damage mechanics model for viscoelastic behavior of composites having microcracks consisting of an isotropic matrix reinforced by independent and inextensible two families of arbitrarily fibers. The elastic stress and strain energy density release rate are expressed in terms of the thermodynamic stress potential, a function of the left Cauchy-Green tensor, the damage tensor, and the fiber distributions. The dissipative stress depends on these quantities and the rate of deformation and the change in time of the damage tensor. The elastic stress and strain energy density release rate and dissipative stress are treated in separate sections. The appropriate invariants used as arguments for thermodynamic stress potential function and dissipative stress function are introduced and the elastic stress, strain energy density release rate, and dissipative stress constitutive equations are worked out. This is followed by specializations for incompressibility, the special case when thermodynamic stress potential is a quadratic polynomial in the invariants and a linearization based on small strains.

The resulting expressions show that the first is attributable to the "inherent" viscoelastic behavior of the undamaged material, while the second is due to the change in time of the damage tensor. As can be noted from constitutive equations for elastic stress, strain energy density release rate, and dissipative stress, the coupling of damage and viscoelasticity introduces very significant complexities in the composite material's response and substantial work remains to be done, both experimentally and analytically, to attain a quantitative and practical understanding of the phenomenon.

In this paper, as a result the constitutive equations relating to the elastic stress, strain energy density release rate, and dissipative stress have been written in terms of material coordinate descriptions. This paper develops a mathematical model based on methods of continuum damage mechanics. After this paper, practical problems will be solved by forming $\mathbf{B}(\mathbf{X})$ and $\mathbf{Z}(\mathbf{X})$ vector fields for various fiber distributions whose parametric equations in the material medium are in the form of $\mathbf{X}=\mathbf{X}(s)$ and necessary interpretations will be made in a more concrete way. Also, in a future work we will study the development of numerical methods for this model.

\section{Conflict of Interests}

The author declares that there is no conflict of interests regarding the publication of this paper.

\section{References}

[1] E.-H. Kim, M.-S. Rim, I. Lee, and T.-K. Hwang, "Composite damage model based on continuum damage mechanics and low velocity impact analysis of composite plates," Composite Structures, vol. 95, pp. 123-134, 2013.

[2] R. S. Kumar and R. Talreja, "A continuum damage model for linear viscoelastic composite materials," Mechanics of Materials, vol. 35, no. 3-6, pp. 463-480, 2003.

[3] R. Talreja, Fatigue of Composite Materials, Technomic Publishing Co., Lancaster, Pa, USA, 1987.

[4] R. W. Sullivan, "Development of a viscoelastic continuum damage model for cyclic loading," Mechanics of Time-Dependent Materials, vol. 12, no. 4, pp. 329-342, 2008.

[5] G. Z. Voyiadjis and B. Deliktas, "A coupled anisotropic damage model for the inelastic response of composite materials," Computer Methods in Applied Mechanics and Engineering, vol. 183, no. 3-4, pp. 159-199, 2000.

[6] G. F. Abdelal, A. Caceres, and E. J. Barbero, "A micro-mechanics damage approach for fatigue of composite materials," Composite Structures, vol. 56, no. 4, pp. 413-422, 2002.

[7] L. Iannucci and J. Ankersen, "An energy based damage model for thin laminated composites," Composites Science and Technology, vol. 66, no. 7-8, pp. 934-951, 2006.

[8] T. E. Tay, G. Liu, A. Yudhanto, and V. B. C. Tan, "A micromacro approach to modeling progressive damage in composite structures," International Journal of Damage Mechanics, vol. 17, no. 1, pp. 5-28, 2008.

[9] P. Maimí, P. P. Camanho, J. A. Mayugo, and C. G. Dávila, “A continuum damage model for composite laminates: part Iconstitutive model," Mechanics of Materials, vol. 39, no. 10, pp. 897-908, 2007.

[10] F. Li and Z. Li, "Continuum damage mechanics based modeling of fiber reinforced concrete in tension," International Journal of Solids and Structures, vol. 38, no. 5, pp. 777-793, 2001.

[11] P. Raghavan and S. Ghosh, "A continuum damage mechanics model for unidirectional composites undergoing interfacial debonding," Mechanics of Materials, vol. 37, no. 9, pp. 955-979, 2005.

[12] J. Lemaitre and J. L. Chaboche, Mechanics of Solids, Cambridge University Press, Cambridge, UK, 1990.

[13] D. Krajicinovic, Damage Mechanics, Elsevier, Amsterdam, The Netherlands, 1996.

[14] G. Z. Voyiadjis and P. I. Kattan, Advances in Damage Mechanics: Metals and Metal Matrix Composites, Elsevier, New York, NY, USA, 1999.

[15] J.-L. Chaboche, "Continuous damage mechanics-a tool to describe phenomena before crack initiation," Nuclear Engineering and Design, vol. 64, no. 2, pp. 233-247, 1981.

[16] Z. X. Wang, J. Lu, H. J. Shi, D. F. Li, and X. Ma, "An updated continuum damage model to investigate fracture process of structures in DBTT region," International Journal of Fracture, vol. 151, no. 2, pp. 199-215, 2008.

[17] J. Li, X.-X. Tian, and R. Abdelmoula, "A damage model for crack prediction in brittle and quasi-brittle materials solved by the 
FFT method," International Journal of Fracture, vol. 173, no. 2, pp. 135-146, 2012.

[18] M. K. Darabi, R. K. Abu Al-Rub, and D. N. Little, "A continuum damage mechanics framework for modeling micro-damage healing," International Journal of Solids and Structures, vol. 49, no. 3-4, pp. 492-513, 2012.

[19] L. M. Kachanov, "Time of the rupture process under creep conditions," Izvestiya Akademii Nauk SSSR. Otdelenie Tekhnicheskikh Nauk, vol. 8, pp. 26-31, 1958.

[20] J. Isometsä and S.-G. Sjölind, "A continuum damage mechanics model for fiber reinforced composites," International Journal of Damage Mechanics, vol. 8, no. 1, pp. 2-17, 1999.

[21] Y. Weitsman, "Continuum damage model for viscoelastic materials," Journal of Applied Mechanics, vol. 55, no. 4, pp. 773-780, 1988.

[22] G. Z. Voyiadjis and P. I. Kattan, "Damage mechanics with fabric tensors," Mechanics of Advanced Materials and Structures, vol. 13, no. 4, pp. 285-301, 2006.

[23] Y. Qiang, L. Zhongkui, and L. G. Tham, "An explicit expression of second-order fabric-tensor dependent elastic compliance tensor," Mechanics Research Communications, vol. 28, no. 3, pp. 255-260, 2001.

[24] A. J. M. Spencer, "Theory of fabric-reinforced viscous fluids," Composites Part A: Applied Science and Manufacturing, vol. 31, no. 12, pp. 1311-1321, 2000.

[25] A. J. Spencer, "A theory of viscoplasticity for fabric-reinforced composites," Journal of the Mechanics and Physics of Solids, vol. 49, no. 11, pp. 2667-2687, 2001.

[26] S. C. Cowin, "The relationship between the elasticity tensor and the fabric tensor," Mechanics of Materials, vol. 4, no. 2, pp. 137$147,1985$.

[27] K.-D. Leng and Q. Yang, "Fabric tensor characterization of tensor-valued directional data: solution, accuracy, and symmetrization," Journal of Applied Mathematics, vol. 2012, Article ID 516060, 22 pages, 2012.

[28] S. Jemioło and J. J. Telega, "Fabric tensor and constitutive equations for a class of plastic and locking orthotropic materials," Archives of Mechanics, vol. 49, no. 6, pp. 1041-1067, 1997.

[29] B. Elmabrouk and J. R. Berger, "Boundary element analysis for effective stiffness tensors: effect of fabric tensor determination method," Computational Mechanics, vol. 51, no. 4, pp. 391-398, 2013.

[30] S. C. Cowin, "Anisotropic poroelasticity: fabric tensor formulation," Mechanics of Materials, vol. 36, no. 8, pp. 665-677, 2004.

[31] K. I. Kanatani, "Distribution of directional data and fabric tensors," International Journal of Engineering Science, vol. 22, no. 2, pp. 149-164, 1984.

[32] E. S. Şuhubi, Continuum Mechanics-Introduction. I.T.U., The Faculty of Arts and Sciences, Istanbul, Turkey, 1994.

[33] M. Usal, M. R. Usal, and A. H. Erçelik, "A constitutive model for arbitrary fiber-reinforced composite materials having microcracks based on continuum damage mechanics," Journal of Applied Engineering, vol. 2, no. 5, pp. 63-81, 2014.

[34] Y. Weitsman, "Damage coupled with heat conduction in uniaxially reinforced composites," Transactions ASME, Journal of Applied Mechanics, vol. 55, no. 3, pp. 641-647, 1988.

[35] A. J. M. Spencer, Deformations of Fibre-Reinforced Materials, Clarendon Press, Oxford, UK, 1972.

[36] A. J. M. Spencer, Continuum Theory of the Mechanics of Fibre-Reinforced Composites, Springer, International Centre for
Mechanical Sciences, Course and Lectures, New York, NY, USA, 1984.

[37] A. C. Eringen, Mechanics of Continua, Robert E. Krieger Publishing, Hungtington, NY, USA, 1980.

[38] A. C. Eringen, Continuum Physics-Mathematics, vol. 1, Academic Press, New York, NY, USA, 1971.

[39] Q.-S. Zheng and A. J. Spencer, "Tensors which characterize anisotropies," International Journal of Engineering Science, vol. 31, no. 5, pp. 679-693, 1993.

[40] Q.-S. Zheng, "On transversely isotropic, orthotropic and relative isotropic functions of symmetric tensors, skew-symmetric tensors and vectors. Part V: the irreducibility of the representations for three dimensional orthotropic functions and the summary," International Journal of Engineering Science, vol. 31, no. 10, pp. 1445-1453, 1993.

[41] A. J. M. Spencer, "Theory of invariants," in Continuum Physics, A. C. Eringen, Ed., vol. 1, pp. 239-353, Academic Press, New York, NY, USA, 1971. 


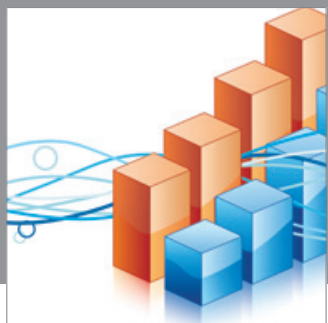

Advances in

Operations Research

mansans

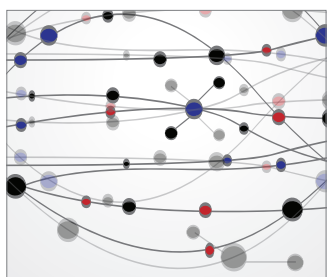

The Scientific World Journal
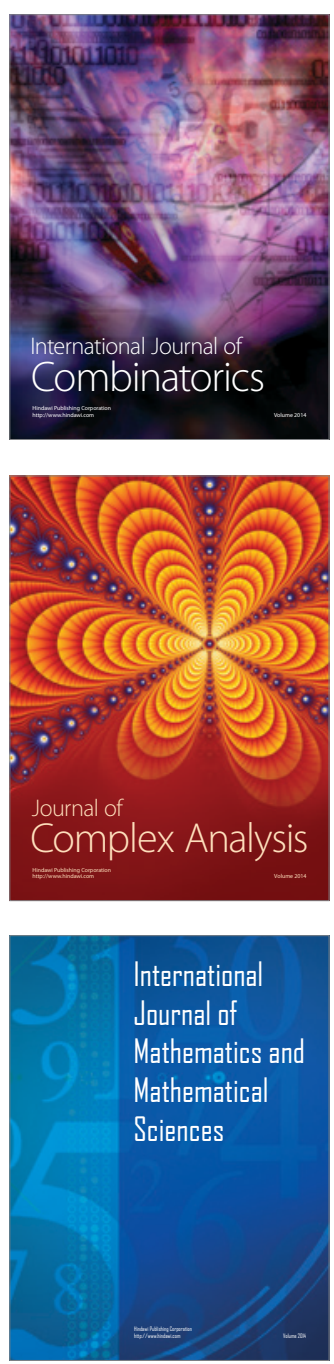
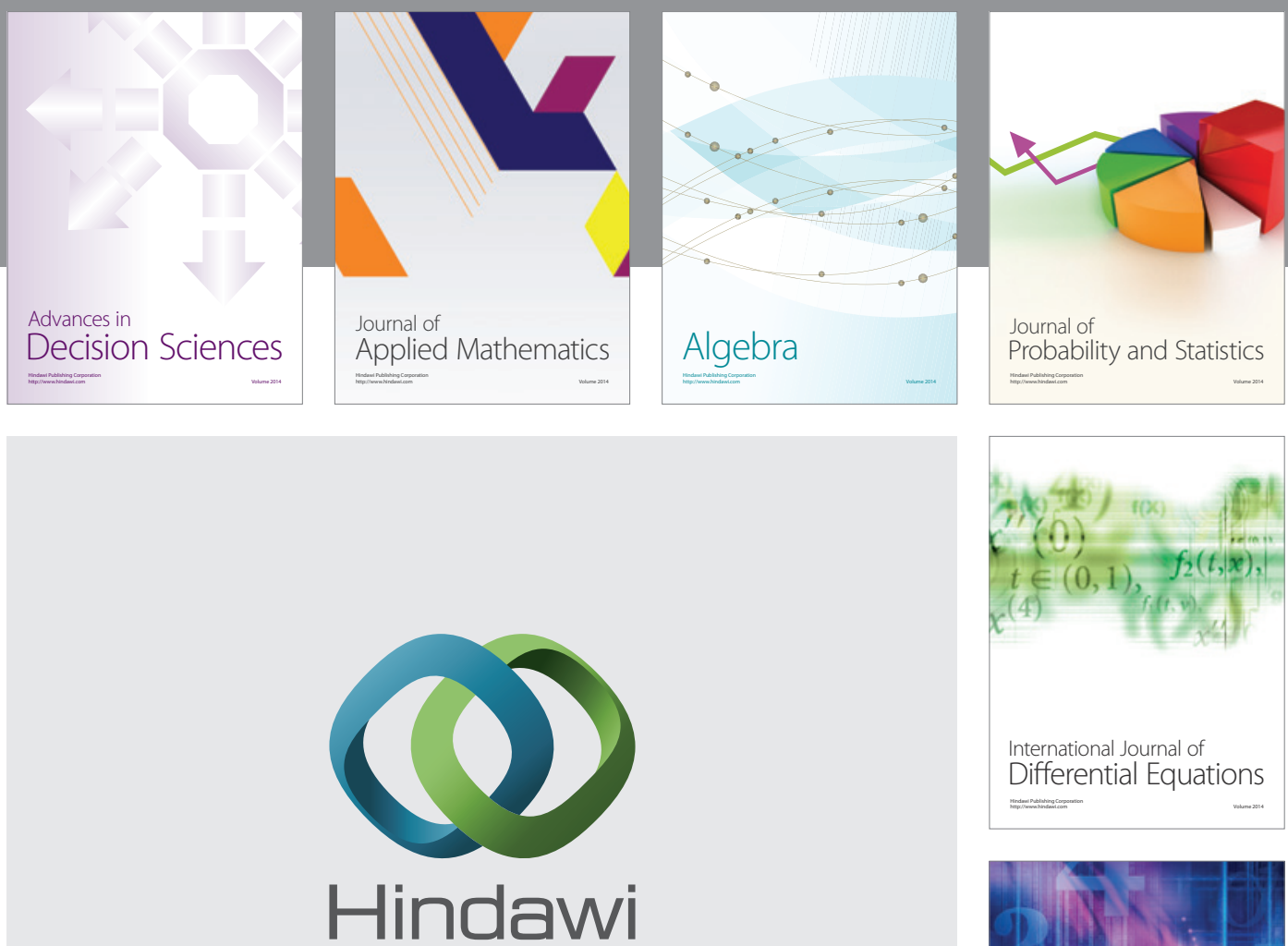

Submit your manuscripts at http://www.hindawi.com
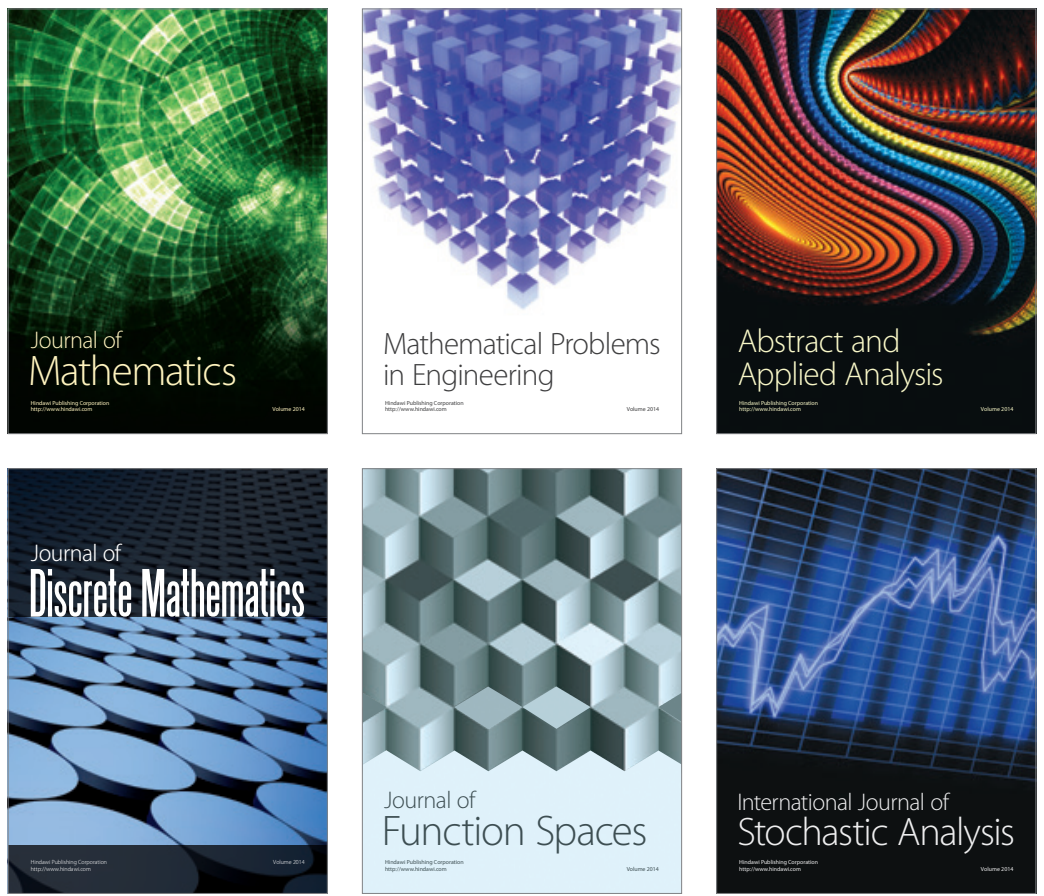

Journal of

Function Spaces

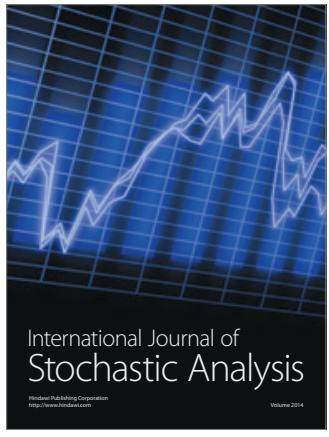

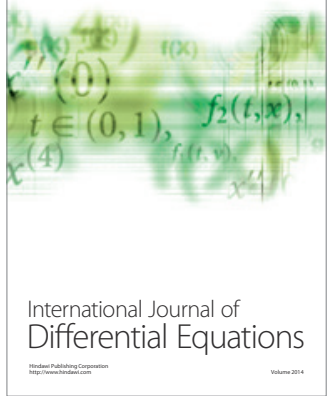
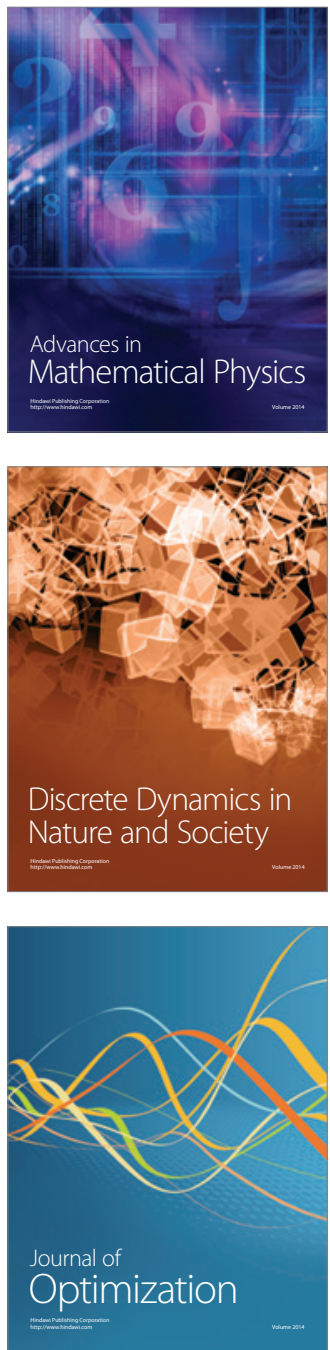